\title{
Sedimentation in Coal-Water Slurry Pipelining
}

\author{
Fabio Rosso
}

Dipartimento di Matematica 'Ulisse Dini’, University of Firenze, Italy

This work was partially supported by the C.N.R. - G.N.F.M. Strategic Project "Metodi Matematici in Fluidodinamica e Dinamica Molecolare" and by the C.N.R. contract \#98.01027.CT01.

\begin{abstract}
In this paper we present an overview of recent investigations on the problem of sedimentation related to the pipelining of a Coal-Water Slurry. The main aspects of the problem are both the determination of the sedimentation velocity, and the understanding and modelling of the dynamics of the sedimentation bed which accumulates on the bottom of the pipe. The analysis is carried out using a combination of suggestions dictated by experimental evidence and suitable mathematical techniques. The result is a model which appears to be both easily manageable and flexible. Predictions of the model are compared with experiments finding a remarkable agreement with the available data.
\end{abstract}




\section{Slurry handling and pipeline transport: a brief outlook on hydro- transport}

Besides intuitive, the idea of transporting solids using a carrier fluid is rather old. Indeed according with Apollodorus and Pausanias ( $1^{\text {st }}$ century B.C.) this transportation technique, nowadays known with the name of Hydrotransport, was first utilized by Herakles, who in one day cleaned up the thirty years' accumulated filth left by thousands of cattle in King Augeas' stables by diverting two rivers to form an open-channel hydrotransport system [42]. Despite of the mythological nature of this tale, known as the fifth Herakles' task, in principle any kind of solid can be moved from a place to another hundreds of miles away using a liquid as transportation tool.

The modern stages of slurry pipeline technology dates back, more or less, to forty years ago. A "slurry"essentially is a suspension of solid particles in a carrier fluid; the interest within these suspensions is that, by using an appropriate technology, they can be pipelined very far away from their production site. Generally the required technology is rather specialized depending on the chemical and physical nature of the suspended particles. This area of investigation is so important that scientists and industries involved within this field meet regularly almost every year at highly specialized international meetings as the Hydrotransport Conferences (thirteen up to now) or the numerous International Conferences on Slurry Technology. The underlying idea is that hydrotransport may be, in many cases, an attractive alternative to other modes of transport (tracks, ships and so on). It also has several advantages like a moderate environmental impact, a relatively little infrastructure work needed and possible low operation and maintenance costs.

For a long time, especially at the early stage of the development of this technology, it was generally thought that the only operational regime to prevent particle settling was the turbulent one; indeed the primary duty when designing a slurry pipeline is to ensure that it will not block because of sediments accumulation on pipe lower wall. However also the cost of maintaining a turbulent regime has to be considered. For this reason recent studies on slurries are addressed towards the possibility to control settling remaining within the laminar regime (which requires less pumping power and is therefore less expensive). If the sediment build-up process could be modeled accurately, then designers would be able to predict the conditions that lead to blockage, and thereby design systems so that the possibility of a blockage is avoided.

The engineering approach to the problem of designing a slurry pipeline is a remarkable combination of basic fluid mechanics and chemical concepts, phenomenological laws, empirical laws, and intuitions guided by specific pilot plant data. The blockage problem is certainly the most focussed one but many others are related with it: the interested reader may take a look to the papers published on this subject in the proceedings of the last Hydrotransport Conferences (see the bibliography at the end). However it is not easy for people not specifically trained in this area to read those contributions and to find out, when looking over a specific problem, a common point of view among different writers. For these reasons, we tried to present an approach to the problem of sediment build-up and evolution of the growing bed which is to a reasonable extent the most self-consistent and readable as possible even for people not expert in this area.

In this paper we report the main results of a research work on the problem of sedimentation in the pipelining of concentrated coal-water slurry (C.W.S.). This subject has involved our group in Industrial Mathematics at the University of Florence for several years. The problem was performed in cooperation with the engineering team of Snamprogetti, an Italian Company well-known among those designing and selling technology for fuel energy production and transportation. In particular Snamprogetti has fully investigated all problems related with C.W.S. technology (see the paper by Terenzi [36] in this same volume) both at their pilot plant in Fano (Italy) and on field, using an industrially operating pipeline ${ }^{1}$. The pilot plant is equipped with sophisticated measuring devices ( like, for example ,a gamma-densimeter) which provide direct evidence and sufficiently clear data on the growth of the bed with time.

Although the model and the approach presented here is dependent on the particular type of slurry under investigation, we believe that the basic ideas can be easily adapted to other similar physical systems.

The C.W.S. mixture considered here enters into the category of concentrated slurries since consists of about $70 \%$ ( by weight) of ground coal such that the size distribution has two peaks around $10 \mu \mathrm{m}$ and $100 \mu \mathrm{m}$. The remaining $30 \%$ is water with a small percentage $(0.5 \%)$ of chemical additive needed to fluidize the suspension. This fluid is perfectly stable at rest even after years, i.e. particle concentration remains everywhere constant in time. This stability is completely to be ascribed to the action of the chemical agent: indeed additive molecules, being highly polar, coat the coal particles with positive charges so that mutual repulsive forces prevent natural sedimentation.

The rheological behaviour of concentrated C.W.S. at low shear rates can be reasonably described by the Bingham model, that is (in laminar flows with simple geometry)

$$
\left(\tau-\tau_{0}\right)_{+}=\eta_{B} \dot{\gamma}
$$

\footnotetext{
${ }^{1}$ Snamprogetti built a $250 \mathrm{Km}$-long operating pipeline connecting Belovo to Novosibinsk (Siberia).
} 
where $\tau, \tau_{0}, \eta_{B}, \dot{\gamma}$ denote the shear stress, the yield stress, the plastic viscosity and the shear rate respectively, and $(\bullet)_{+}$ means the positive part of $(\bullet)$. Of course more complicated models can be used but (1) is sufficient for our purposes.

When a C.W.S. is stirred in a vessel or pumped through a pipeline, the action of shear dramatically modifies this picture through a phenomenon called rheological degradation which has also been deeply investigated and fully explained (see, for example, [9],[10],[11],[15],[21], [22],[23], [24], [26], [32]). However the time scale of this phenomenon is many orders of magnitude larger than that of sedimentation, so that if we focus on the latter problem, the time dependency of rheological parameters can be neglected. Therefore we can consider a C.W.S. as a time-independent Bingham fluid.

The solid fraction does not generally consist only of pure coal and even after a suitable treatment (beneficiation), ground coal from the mill hardly contains less than about $6 \%$ of other micronized minerals and steel residues due to beneficiation itself.

Impurities have generally a size comparable with or higher than that of the top size of coal particles; the inner structure of a C.W.S. provided by the residual adhesion forces among the coal particles (which is responsible for the yield stress) is sufficient to prevent settling at rest. This is no longer true when a CWS experiences a shear rate, as if it is stirred in a viscometer of pumped in a pipeline. Indeed while the tendency to settle of the pure coal particles is prevented by the action of the chemical stabilizer, this is not true for impurities which, having a different chemical structure, do not react with the additive. Therefore the settling of these particles cannot be controlled, leading to a sediment build-up at the bottom of the pipe when the CWS is pipelined at long distances. This phenomenon can also be observed if the CWS is placed in a rotating bob-cup viscometer. In the pipe flow the reduction of the hydraulic diameter due to the sediment build-up leads either to increase the pressure gradient (assuming that we can) in order to maintain constant the discharge or to decrease the latter if we can not increase the former. Both cases are either unsafe or economically disadvantgeous. Therefore what is needed is a model to predict the evolution of the growing bed in order to choose the optimal discharge and to plan the periodical (unavoidable) shut-down of the pumps and cleaning of the first portion of the pipeline (the only one interested to the settling phenomenon).

The aim if this article is just to describe the work of investigation during last years about the above problem using the facilities of Snamprogetti, and the results of this effort.

\section{Some general facts about sedimentation}

Consider a single spherical rigid particle settling with constant velocity $\mathbf{v}_{s}$ in a Newtonian viscous liquid at rest and which extends to infinity in all directions. The uniform motion of the falling particle results from the equilibrium of three forces: the particle's own weight $\frac{4}{3} \pi \delta^{3} \rho_{s} \mathbf{g}$, Archimedes' lifting force $-\frac{4}{3} \pi \delta^{3} \rho_{l} \mathbf{g}$, and the viscous force exerted by the fluid on the sphere. For small Reynolds numbers (say $\mathcal{R} e \ll 1$ ) Stokes [35] proved that the viscous force exerted by the fluid (drag force) is parallel (and opposite in direction) to $\mathbf{v}_{s}$ and of magnitude $6 \pi \delta \eta v_{s}$. Then a force balance immediately yields the well known formula

$$
v_{s}=\frac{2 g\left(\rho_{s}-\rho_{l}\right)}{9 \eta} \delta^{2} .
$$

It is a common practice to express the forces exerted on moving bodies by the fluid in terms of a dimensionless parameter $C_{D}$ called drag coefficient obtained by dividing the drag force $6 \pi \delta \eta v_{s}$ by $(1 / 2) \rho_{s} v_{s}^{2}$ and by the area of the body projected on a plane normal to $\mathbf{v}_{s}$; thus the drag coefficient is here

$$
C_{D}=\frac{24}{\mathcal{R} e}, \quad \text { with } \mathcal{R} e=\frac{2 \delta v_{s} \rho_{s}}{\eta}
$$

For a particle of sand falling through water at room temperature, the hypothesis $\mathcal{R} e \ll 1$ is satisfied provided that $\delta \ll 0.006 \mathrm{~cm}$. From the mathematical point of view, the Stokes's hypothesis $(\mathcal{R} e \ll 1)$ is equivalent to a suitable linear approximation of the nonlinear Navier-Stokes equation. This approximation (and consequently the Stokes's solution) contains various drawbacks and physical inconsistencies (the most famous one is probably the so-called Stokes Paradox, see for example [25]). Oseen [30][31] proposed a different linearization which yields, instead of (3), the following expression

$$
C_{D}=\frac{24}{\mathcal{R} e}\left(1+\frac{3}{16} \mathcal{R} e\right) .
$$

However, in many cases of practical interest, both (3) and (4) are not in agreement with measured values. On the base of experimental evidence, a variety of "ad hoc"formulas for the terminal velocity of the particle have been suggested; a relatively recent state of art can be found in [13] and [14]. Research in this field, being based almost exclusively on sophisticated experiments and numerical simulations, is still very active. 
In a typical sedimentation problem we have a situation rather different from that considered theorically: the host fluid may be non-newtonian and may be sheared, particle settles within a dense population of similar particles, particles' shape and volume are are irregular and randomly distributed, particle surface may change its electrical charge distribution interacting with chemical additives present in the host fluid, wall effects on falling particles may not be negligible, and so on.

Cases in which the host fluid is non-Newtonian have been extensively studied in recent years. Several theoretical and/or experimental approaches have been used and suggestions have been made to correct the correlation (3) for the different rheological behaviours. For fluids without yield stress ( in particular the Power Law fluids), one can refer to Acharya et al.[1], Leal [27] and to the review papers of Chhabra [12] and [13], where an rather complete bibliographical information can be found.

For Bingham fluids the situation is more complicated; here we give a short account of the previous investigation, referring to [13] for further information and details.

First of all the yield stress $\tau_{0}$ can prevent settling of particles up to a critical radius which, if $\Delta \rho:=\rho_{s}-\rho_{l}$, is given by

$$
\delta_{0}=\alpha \frac{\tau_{0}}{g \Delta \rho},
$$

as it can be seen, simply assuming that the force due to the yield stress is proportional to it and that to the area of the spherical particle.

Concerning the numerical value of the proportionality constant $\alpha$ there is no agreement among different authors, $\alpha$ ranging from 1.5 to 10 ; these differences however can be mainly ascribed to possible different (being experimentally very delicate) determinations of $\tau_{0}$.

The study of the motion of a spherical particle in a Bingham plastic fluid has also been investigated both from a theoretical and experimental point of view; we recall the papers by Tyabin [38] and Andres [3] where the drag force is expressed as

$$
F_{D}=\alpha \delta^{2} \tau_{0}+\beta \eta_{B} v_{s} \delta
$$

where the coefficients $\alpha$ and $\beta$ are either experimentally fitted or theoretically calculated in terms of the quantity $k:=\left(3 \tau_{0} /(\delta g \Delta \rho)\right)^{1 / 2}$. In subsequent papers a correlation between $C_{D}$ and a modified Reynolds number, was outlined (the interested reader may consult the papers of Ansley and Smith [4], and Dedegil[19]). Other interesting contributions on this subject are due to [39], [40], and [41].

A very good fit with the above experimental results was obtained by Beris et al. [6] integrating the equation of motion by finite elements, thus also recovering the lower and upper estimates previously obtained by Yoshioka et al. [43].

In all the above papers the motion of a single sphere with a sufficiently large radius in an unsheared environment was considered. We also mention the paper by Thomas [37] where an experiment of sedimentation in a sheared fluid was described.

As we have seen, the general picture concerning sedimentation is rather involved and there is no conclusive theory flexible enough to be adapted to a variety of settling phenomena in pipeline flows.

In [15] and [17] we presented some new ideas about the problem of determining $v_{s}$ in a sheared fluid (not necessarily Newtonian), when the particle belongs to a large settling population. Of course in a sheared environment the particle velocity is now expected to depend on the local value of the shear rate. The novelty of our approach consists mainly in looking at the problem of finding the effective viscosity experienced by a population $\mathcal{P}$ of particles as an inverse problem.

Indeed a possible alternative approach to the problem of finding the correct form of $v_{s}$ could be be the following: to collect data (generally a discrete set) from some suitably designed experiment on the base of some formula of type

$$
\text { data }=\mathcal{F}\left(v_{s}\right),
$$

where $\mathcal{F}$ is determined by the geometry and the type of experiment. If we don't make any a priori assumption about the form of $v_{s},(7)$ is an inverse problem with discrete data to which we could apply the technique developed by Bertero, De Mohl and Pike some years ago (see [7] and [8]). In the Appendix we give a short presentation of this functional method. We tested this approach against available experimental data and we found encouraging conclusions. However the method is specifically designed for laboratory tests and cannot be directly applied to a Bingham flow in a pipeline. Nevertheless, we can derive useful indications to deduce the settling velocity of a particle as a function of the radial coordinate of the pipe, if the flow is stationary.

In the next section we present the derivation of $v_{s}$ with particular reference to C.W.S. 


\section{Sedimentation in a CWS sheared in a rotating viscometer}

The CWS used in the experiments considered to collect our data was prepared with a Polish coal according to SP REOCARB process (a Snamprogetti patent) ${ }^{2}$

The geometry we considered is that of a rotational bob-cup viscometer (designed on purpose with a widened gap), where the settling of particles is transverse to the direction of shearing (exactly as in the pipeline flow). The viscometer was filled up with CWS in which a population $\mathcal{P}$ of sand particles with density $\rho_{s}>\rho_{C W S}$ has been initially homogeneously dispersed.

Then we pointed our attention over a cross-sectional cell $C_{z}$ of the viscometer of thickness $h$, with top and bottom bases placed respectively at $z-h$ and $z$, the $z$-axis being directed as the gravity vector $\mathbf{g}$. The quantity to be measured is the net mass variation in $C_{z}$ due to the settling of $\mathcal{P}$.

Let us first notice that there may be a fraction of $\mathcal{P}$ which does not settle, depending on the steady laminar shearing motion generated by a uniform rotation of the inner cylinder. This circumstance has to be ascribed to the internal structure of the CWS (responsible for the yield stress), which is partially or totally modified by the shearing conditions. Indeed in Bingham fluids at rest the yield stress may be able to sustain suspended particles (see, for example, the article [36] in this book).

For a fluid in laminar motion in a rotational viscometer, $\dot{\gamma}$ denotes the absolute value of the only significant component $\frac{d u}{d r}$ (with $\mathbf{u}=(0, u(r), 0)$ ) of the strain rate tensor.

We do not postulate the existence of a relationship of the type (5) nor enter the debate about the dimension and shape of the unsheared envelope surrounding the particles hypothized by some Authors (see [4],[2][6] and [14] for a review on this topic).

Better references for experiment details concerning the theory we are going to illustrate, are [15] and [36]. We confine ourselves to report only the main conclusions.

In a Bingham plastic there can be, in principle, a fraction of $\mathcal{P}$ which does not settle, depending on the particle size, on $\tau_{0}$, and on $\dot{\gamma}$. If we call $\delta_{0}$ the critical value of $\delta$ below which particles do not settle, the critical radius - shear rate curve can be evaluated from experimental data by measuring the fraction of $\mathcal{P}$ which remains in the upper cell of observation at the latest shearing time. The function $\delta_{0}(\dot{\gamma})$ can be determined using the law

$$
\mathcal{M}_{\infty}=\mathcal{M}_{0} \frac{\int_{0}^{\delta_{0}(\dot{\gamma})} \Omega(\delta) d \delta}{\int_{0}^{\infty} \Omega(\delta) d \delta},
$$

where $\mathcal{M}_{\infty}$ is the mass of particles retained in $C_{1}$ (the highest observation cell) for $t \rightarrow \infty, \Omega(\delta)$ is the particle radius distribution function per unit mass of $\mathcal{P}$ and $\mathcal{M}_{0}$ is the mass of particles present in $C_{1}$ at $t=0$.

Dimensional analysis shows that particles starting with zero velocity reach the stationary settling regime almost instantaneously. Thus each cell $C_{z}$ will experience an emptying wave starting from the moment in which the heaviest particle, which left the level $z=0$ at $t=0$, has reached the level $z-h$.

The settling velocity of a particle of radius $\delta$ is assumed to be of the form

$$
v_{s}\left(\alpha ; \delta, \delta_{0}\right)=\alpha f\left(\delta, \delta_{0}\right),
$$

where $f\left(\delta, \delta_{0}\right)$ is an increasing function of $\delta\left(\delta \geq \delta_{0}\right)$, such that $f\left(\delta_{0}, \delta_{0}\right)=0$, and the parameter $\alpha$ has to be determined as a function of $\dot{\gamma}$.

In the spirit of Stokes's formula (2), we can introduce an effective viscosity for sedimentation

$$
\eta_{e f f}:=\frac{1}{\alpha}
$$

In the classical Newtonian case we would have $\delta_{0}=0, f(\delta)=\delta^{2}$, and $\eta_{\text {eff }}$ is just, up to a proportionality constant, the fluid bulk viscosity $\eta$.

As far as the choice of the function $f$ is concerned, we follow Dedegil [19] who proposed

$$
f\left(\delta, \delta_{0}\right)=\delta\left(\delta-\delta_{0}\right)_{+} ;
$$

however we think of $\delta_{0}$ as given by the experimental formula (8), better than (5).

\footnotetext{
${ }^{2}$ All laboratory experiments concerning the settling of particles in a rotating viscometer were performed and monitored by Snamprogetti.
} 
Via an elementary mass balance it easy to see that the mass variation experienced by $C_{z}$ is given explicitly by

$$
\mathcal{M}(\alpha ; t, \dot{\gamma})=\mathcal{M}_{0}+\rho_{s} \Sigma \mathcal{K} \int_{\delta_{0}}^{\infty} \Omega(\delta) \Phi\left[v_{s}(\delta) ; t\right] d \delta,
$$

where $\Sigma$ is the cross sectional area of $C_{z}, \mathcal{K}$ is the volume fraction of $\mathcal{P}$ to the mixture (CWS plus $\left.\mathcal{P}\right)^{3}$, and

$$
\Phi\left[v_{s}(\delta) ; t\right]=\min \left\{v_{s}(\delta) t, z-h\right\}-\min \left\{v_{s}(\delta) t, z\right\} .
$$

For every cell $\mathrm{C}$ and for every fixed pair shear rate $\dot{\gamma}$ - shearing time $t$, the left hand side of (10) is a number given by the experimental data and the right hand side of (10) is just an implicit function of $\alpha$. Solving numerically such equation we obtain the corresponding values of the parameter $\alpha$. We expect, within the limits of the experimental errors, that such parameter $\alpha$ does not depend on the cell of observation as well as on the shearing time $t$. Therefore we can define a function $\eta_{e f f}=\eta(\dot{\gamma})$. Using equation (9), we can compare the experimental data with the emptying wave (10) and we obtain the corresponding values of $\alpha$ as a function of $\dot{\gamma}$ (see figure 1). In all experiments worked out at Snamprogetti laboratories $\delta_{0}(\dot{\gamma})$ turned out to be practically zero. Therefore we definitely set $f(\delta)=\delta^{2}$ and consequently

$$
v_{s}(\dot{\gamma}, \delta)=\alpha(\dot{\gamma}) \delta^{2}
$$

The knowledge of the function $\alpha(\dot{\gamma})$ is crucial to develop the model for the evolution of the sedimentation bed in a pipeline, which will be presented in the next section.

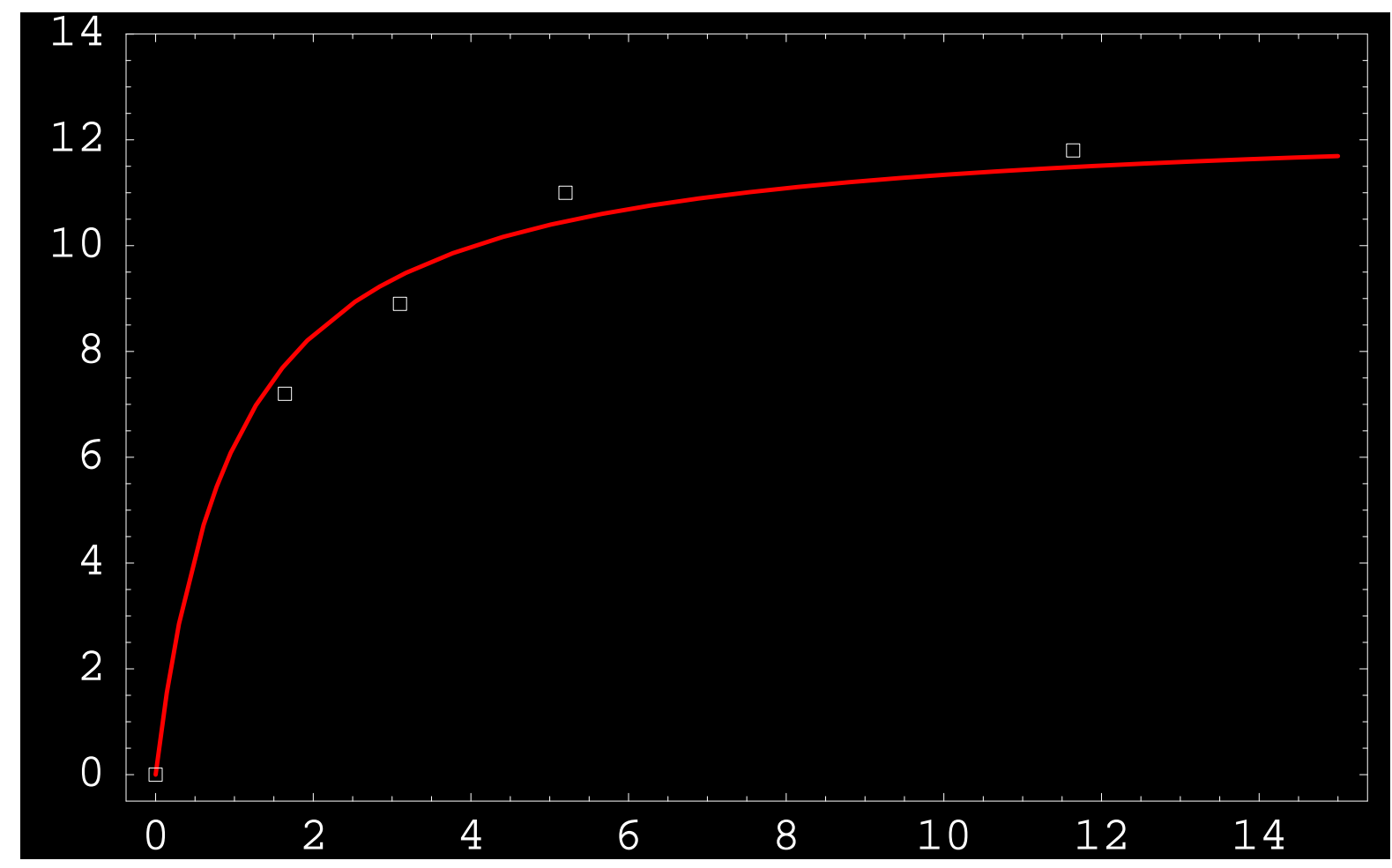

Figure 1: Experimentally determined values of $\alpha(\dot{\gamma})$ for $\dot{\gamma}=\{0.001,1.35,2.51,4.64,11$.$\} ; the solid line is the graph of 12.4103-$ $12.3487 e^{-x / 2}$, a nonlinear fit of these data via the Least Square Method.

\section{Build-up and evolution of the sedimentation bed in a pipeline}

The argument of this Section is largely based on the ideas presented in [16],[20], [28], and [29]. Let us first specify the geometry (see figure 2 and symbols.

\footnotetext{
${ }^{3}$ By definition $\mathcal{K}=\frac{4 \pi}{3} \int_{0}^{\infty} \delta^{3} N(\delta) d \delta$ where $N$ denotes the size distribution function of $\mathcal{P}$ per unit volume of mixture $(\mathcal{P}+C W S)$. Therefore $N(\delta)=\rho_{s} \mathcal{K} \Omega(\delta)$.
} 


\section{Sections of the pipeline}
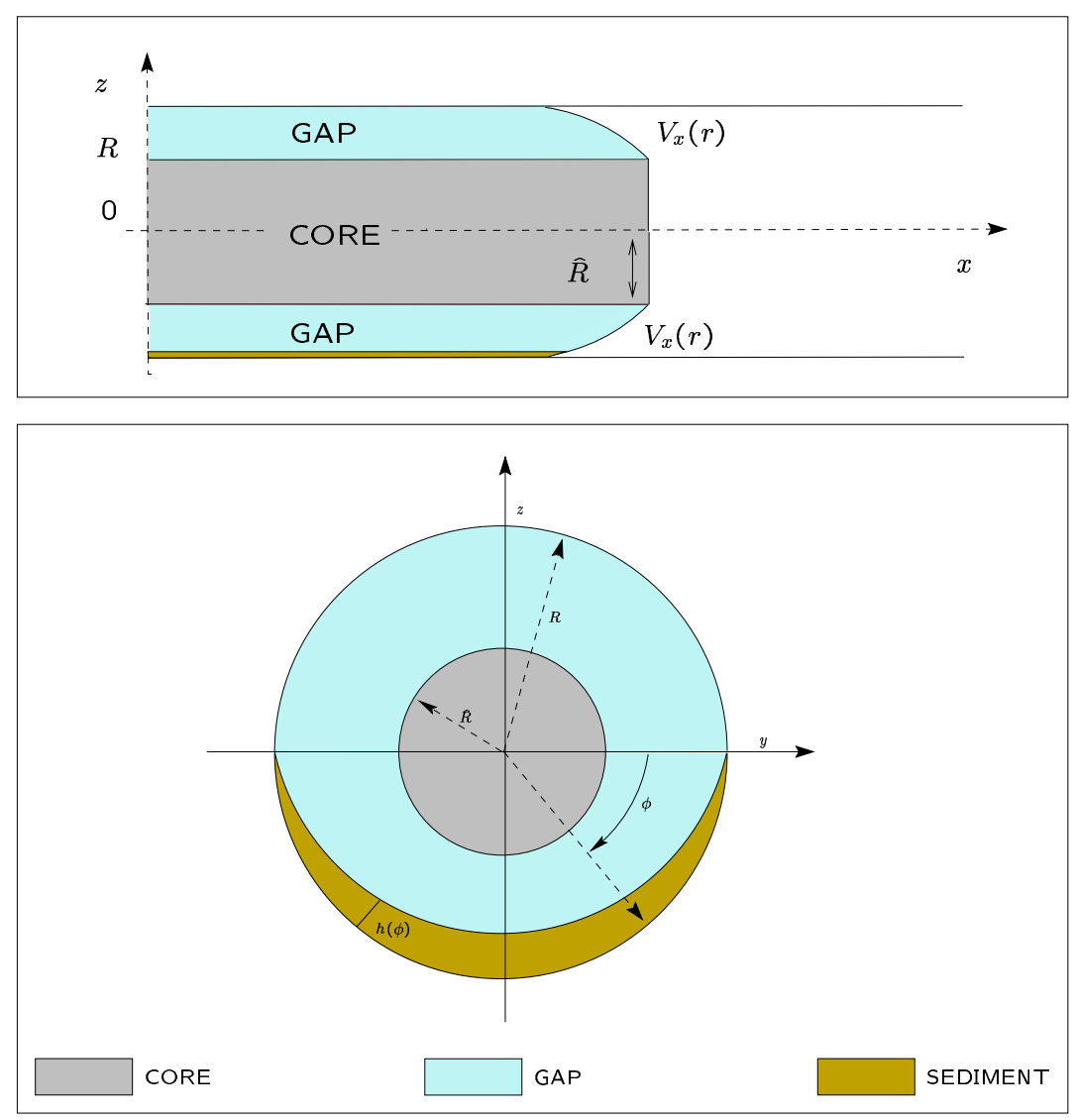

Figure 2: Longitudinal and transversal cross-sections of the pipe; the function $h(x, t, \phi)$ describes the bed profile (the indicated profile is only for illustration purposes)

If $\frac{d p}{d x}$ denotes the constant pressure gradient $(<0)$ and $V_{x}(r)$ the velocity profile of the main flow, $\dot{\gamma}=\dot{\gamma}(r)=$ $\left|\frac{d V_{x}}{d r}\right|$ is the shear rate; as we said in Section 1 the C.W.S. behaves as a Bingham fluid, therefore we still assume 
$\left(\tau-\tau_{0}\right)_{+}=\eta_{B} \dot{\gamma}$. Then, by coupling the Navier-Stokes equations with boundary conditions, one easily gets

$$
V_{x}(r)= \begin{cases}-\frac{R^{2}}{4 \eta_{B}} \frac{d p}{d x}\left(1-\frac{r^{2}}{R^{2}}\right)-\frac{\tau_{0} R}{\eta_{B}}\left(1-\frac{r}{R}\right), & \text { for } \quad r \geq \hat{R}, \\ -\frac{1}{4 \eta_{B}} \frac{d p}{d x}(R-\hat{R}), & \text { for } \quad r \leq \hat{R},\end{cases}
$$

where $r=\hat{R}$ is the interface bounding the rigid core. The momentum balance equation implies $\tau=\frac{r}{2}\left|\frac{d p}{d x}\right|$, so that

$$
\hat{R}=\frac{2 \tau_{0}}{|d p / d x|}
$$

moreover

$$
\dot{\gamma}(r)=\frac{1}{2 \eta_{B}}\left|\frac{d p}{d x}\right|(r-\hat{R})_{+} .
$$

A key assumption of the present model is that the sediment thickness $h$ is sufficiently small compared to the pipe radius $R$ so that the flow geometry will never be significantly affected by the sedimentation bed growing at the bottom of the pipe. Bearing in mind that for values of $h$ close to $2 \%$ of the pipe diameter a precautional shut down and cleaning operation is mandatory, the above assumption does not sound as a limitation.

The trajectory of a particle $P=P\left(\delta, \rho_{s},\left[0, y_{0}, z_{0}\right]\right)$ of radius $\delta$, density $\rho_{s}$ entering the pipe at the initial position $\left(0, y_{0}, z_{0}\right)$ is fully described by the system

$$
\left\{\begin{array}{l}
\dot{x}=V_{x}(r), \\
\dot{z}=-v_{s}(\delta, r), \\
r=\sqrt{z^{2}+y_{0}^{2}} .
\end{array}\right.
$$

Recalling the analysis of Section 3, we assume the settling velocity $v_{s}$ to be $v_{s}(\delta, r)=\alpha(\dot{\gamma}(r)) \delta^{2}$. The function $\alpha(\dot{\gamma})$, determined experimentally ${ }^{4}$, is that shown in figure 1 .

It is quite evident from the above system that particle motion remains confined in the vertical plane $y=y_{0}$. Moreover experiments definitely show that $\alpha(0)=0$ and $\dot{\alpha} \geq 0$. As a consequence only particles incoming the pipe through the gray-shaded region shown in figure 3 do contribute to the growing sediment.

\footnotetext{
${ }^{4}$ We have a numerical code which gives $\alpha(\dot{\gamma})$ on the base of laboratory tests in which the settling phenomenon is suitably monitored in a bob-cup viscometer as described in Section 3.
} 


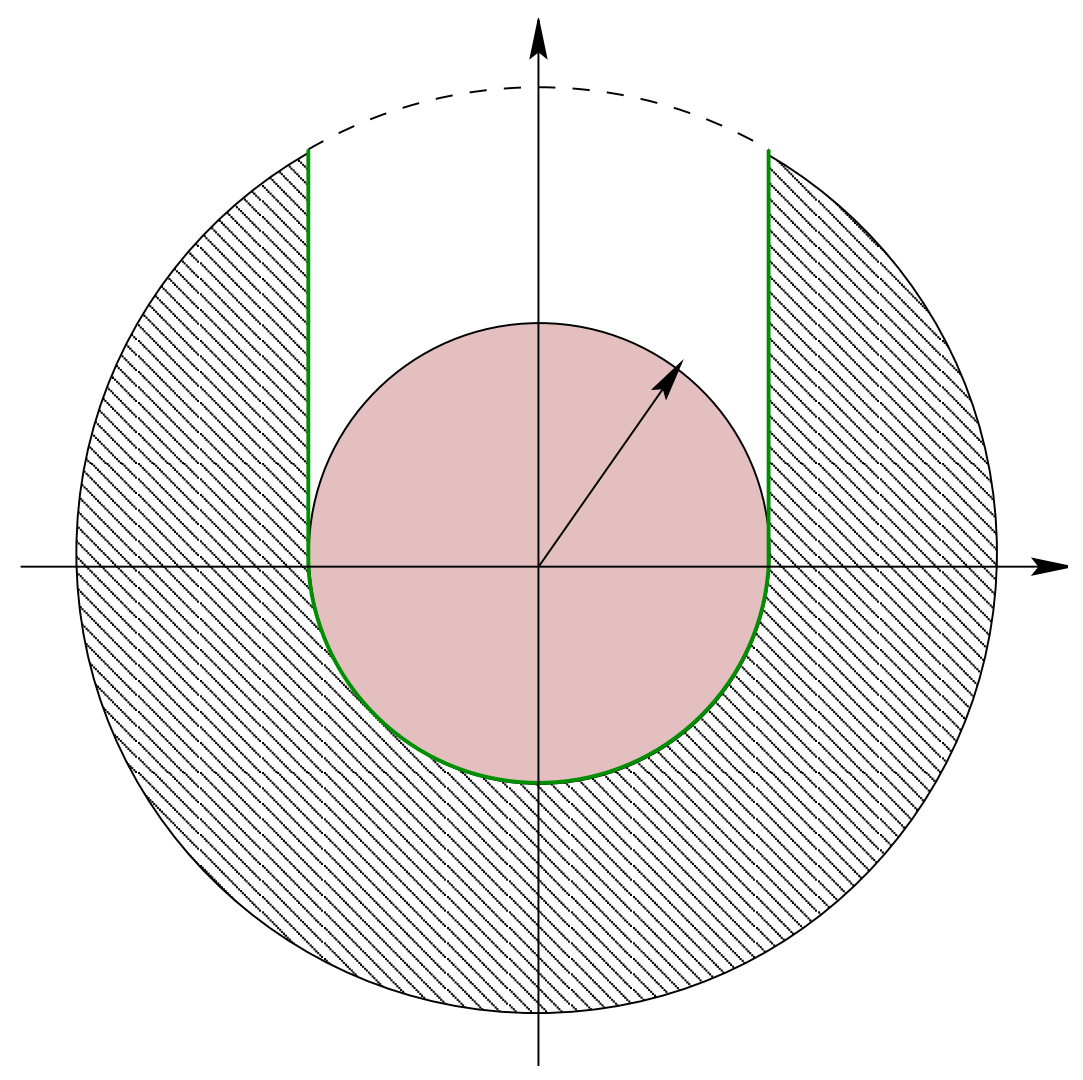

Figure 3: Transverse cross section of the pipe with contributing and noncontributing regions to the dynamics of the bed. In the darker central region particles do not settle since $\dot{\gamma}=0$ while in the white region above they settle but never reach the bed; only particles settling in the shaded reagion contribute to the growing of the bed.

Any particle $P$ incoming through the gray-shaded region of the initial cross-section $\{x=0\}$ ends its trajectory at the point $\left(x^{\star}, y_{0}, z^{\star}\right)$ defined via

$$
\left\{\begin{aligned}
z^{\star}= & -\sqrt{R^{2}-y_{0}^{2}}, \\
x^{\star}= & \int_{z}^{z_{0}} \frac{V_{x}(\tilde{r})}{v_{s}(\tilde{r}, \delta)} d \tilde{z}, \\
& \text { with } \tilde{r}=\sqrt{\tilde{z}^{2}+y_{0}^{2}} .
\end{aligned}\right.
$$

From (17) it can be easily proved that, for any fixed $x^{\star}$, the locus $\Gamma\left(x^{\star}, \delta\right)$ of points on $\{x=0\}$ formed by those particles with radius $\delta$, ending their trajectories on the pipe wall at $x=x^{\star}$, is actually a graph (see figure 4) which we denote by

$$
z_{0}=\mathcal{Z}\left(y_{0} ; x^{\star}, \delta\right)
$$




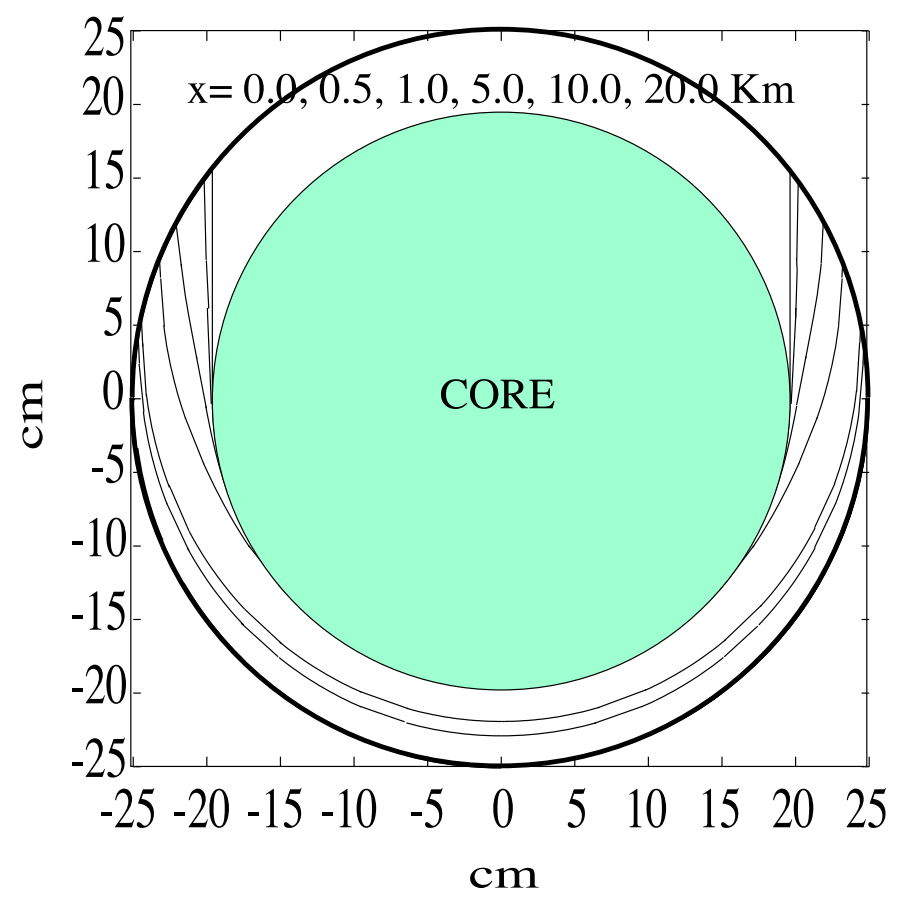

Figure 4: Some $\Gamma\left(x^{\star}, \delta\right)$ graphs for $Q=250 \mathrm{~m}^{3} / \mathrm{h}, \delta=0.0067 \mathrm{~cm}$.The slopes of the $U$-shaped curves increase with $x^{\star}$. The steepest $U$-shaped curve corresponds to $x^{\star}=+\infty$

The settling rate per unit length at distance $x$ from the initial cross-section due to particle with radii between $\delta$ and $\delta+d \delta$ is given by

$$
S(x ; \delta) d \delta=\frac{4}{3} \pi \delta^{3} \rho_{s} N(\delta) d \delta \int_{-y_{M}}^{+y_{M}} v_{s}\left(\sqrt{y_{0}^{2}+(\mathcal{Z})^{2}}, \delta\right) d y_{0},
$$

where $\mathcal{Z}=\mathcal{Z}\left(y_{0} ; x, \delta\right), N(\delta) d \delta$ is the number of settling particles with radii between $\delta$ and $\delta+d \delta$ per unit volume of mixture $(\mathcal{P}+C W S)$, and the endpoints $\pm y_{M}$ of $\Gamma(x, \delta)$ are implicitly defined by

$$
y_{M}^{2}(x, \delta)+\mathcal{Z}^{2}\left(x^{\star}, y_{M}(x, \delta)\right)=R^{2} .
$$

Since $N$ vanishes outside $\left[\delta_{\min }, \delta_{\max }\right]$, the overall settling rate (per unit length of the pipeline) will be given by

$$
S_{T}(x)=\int_{\delta_{m}}^{\delta_{M}} S(x ; \delta) d \delta .
$$

Analysis shows that $S_{T}$ is a rapidly decreasing function of $x$ and is practically zero if $x$ is sufficiently large ${ }^{5}$.

\footnotetext{
${ }^{5}$ In our simulations with a known population of sand particles $\left(\delta_{\min }=0.0035 \mathrm{~cm}, \delta_{\max }=0.0113 \mathrm{~cm}\right.$, see figure 5$), \rho_{s}=2.67 \mathrm{~g} / \mathrm{cm}^{3}$ and with a CWS with known rheological characteristic parameters $\left(\tau_{0}=8.89 P, \eta_{B}=0.16 P s\right.$ we found $S_{T} \simeq 0$ at $x=10 K m$ for a flow rate $Q$ of $\simeq 100 \mathrm{~m}^{3} \mathrm{~h}^{-1}$ and at $x=60 \mathrm{Km}$ for a flow rate $Q$ of $\simeq 450 \mathrm{~m}^{3} \mathrm{~h}^{-1}$ (pipe radius $R=25 \mathrm{~cm}$.
} 


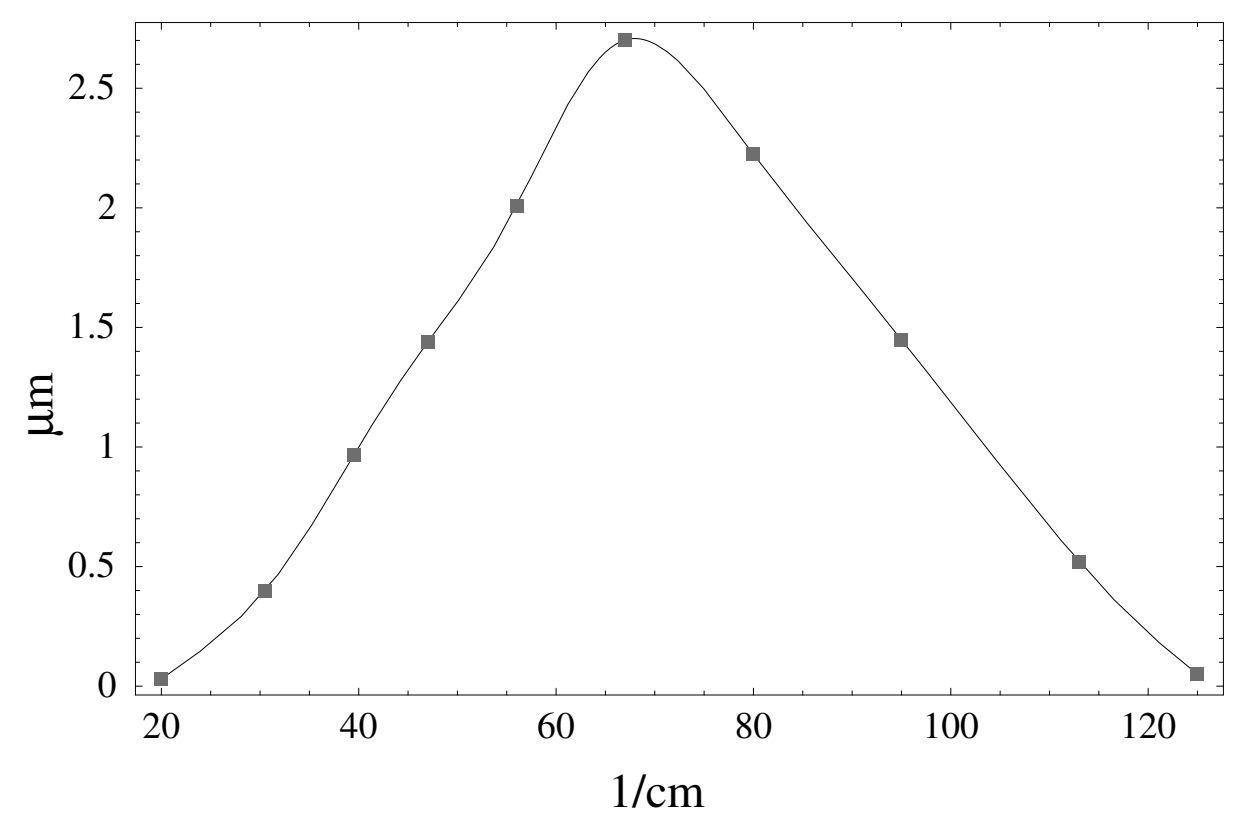

Figure 5: Size distribution of sand particles used for experiments: the continuous line is a spline fit of data points $\left(\delta, \delta^{3} N(\delta)\right)$ (units are $(\mu m, 1 / \mathrm{cm})$ ).

Equation (19) provides the source term balancing the rate of change of the cross-sectional area $a(x, t)$ of the bed. However a precise and complete description of the actual dynamics of the bed appears to be rather complex. Indeed there are infinitely many different profiles $h(\phi, x, t)$ which correspond to the same function $a(x, t)$ and there is no "natural"equation to describe the evolution of $h$. However such level of sophistication is absolutely not needed in our case, since for the particular nature of the problem, experimental observations cannot be that accurate. Indeed the best equipment available (a gamma-densimeter) provides only a reasonable measure of the average thickness of the bed as a function of time where the monitoring device is placed. The only clear experimental indication is that pure settling cannot be the only driving mechanism in the dynamics of the bed. Indeed, were this the case, the bed should grow continuously, filling the pipe in a finite time. Therefore pure settling (fully described by $S_{T}(x)$ ) needs to be coupled with a transport action, which consists in a partial mass removal in the horizontal direction due to the action of the main flow. The idea is that, during a first stage, the whole bed flows in the pipe; then, when the bed has reached a critical thickness $\Delta$, a static layer begins to grow just below the dynamic one (see figure 6). The value of $\Delta$ depends on the main volumetric flow rate $Q$. 


\section{Pure Settling}
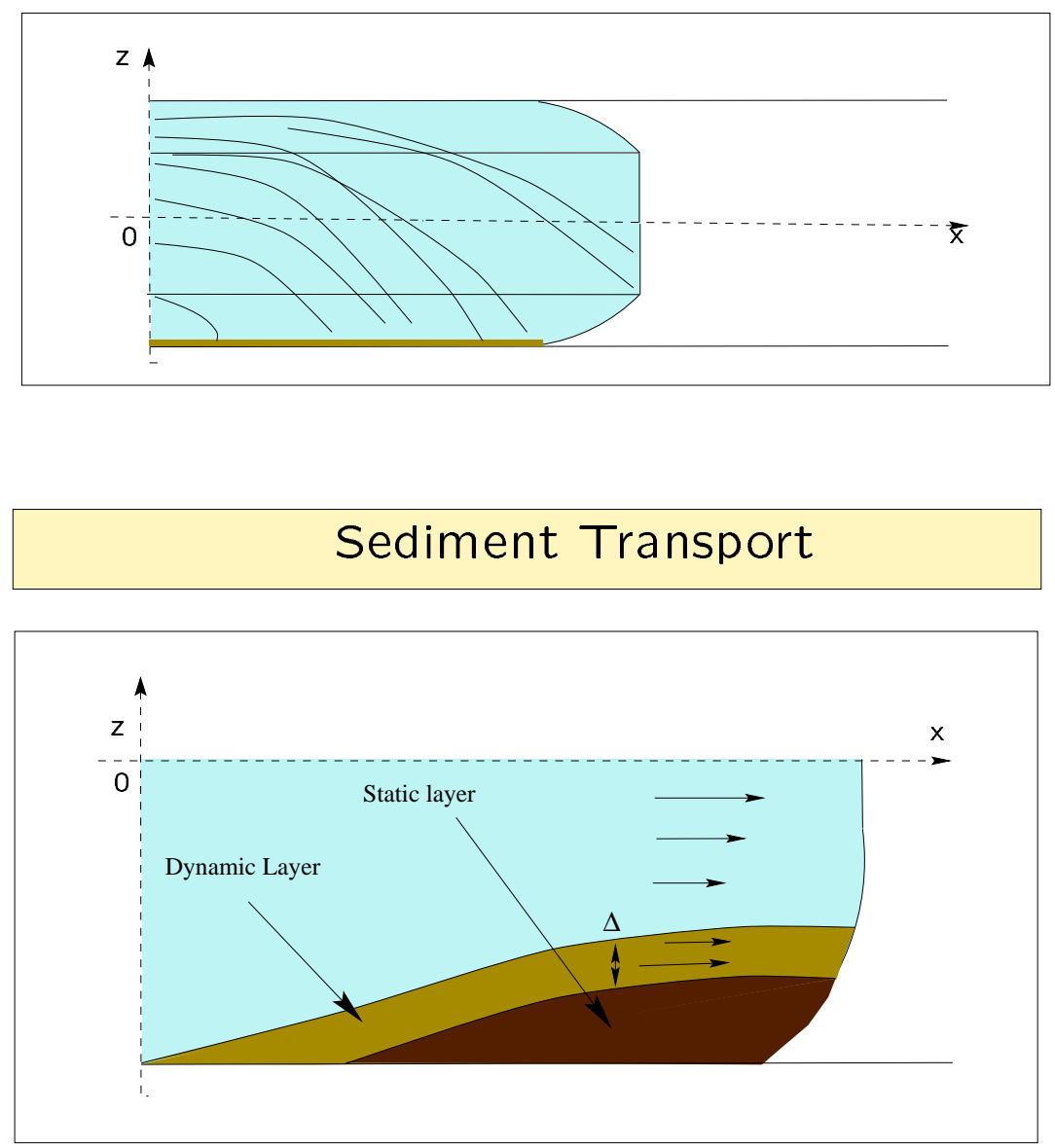

Figure 6: The settling and transport actions driving the dynamics of the bed; when the dynamic layer has reached the critical thickness $\Delta$, the static sublayer begins to develop below it.

To be more precise, the model is based on the following hypotheses:

(i) the radial thickness $h(\phi, x, t)$ of the bed (see figure 7) is proportional to its cross-sectional area $a(x, t)$ via

$$
h(\phi, x, t)=C(\phi) a(x, t)
$$

(ii) the cross-sectional area of the bed remains always small enough so that the flow geometry of the main flow is not significantly modified (in [33] is partially relaxed); in other words 


$$
a(x, t) \ll \pi R^{2} .
$$

(iii) The cross-sectional profile is sufficiently regular and physically consistent in the sense that $C(\phi)$ must satisfy the following constraints:

1. $C(0)=C(\pi)=0$,

2. $C^{\prime}(\phi)>0$, for all $\phi \in[0, \pi / 2)$,

3. $C(\phi)=C(\pi-\phi)$, for all $\phi \in[0, \pi / 2)$,

4. $C^{\prime}(\phi) \leq\left[\frac{R}{a(x, t)}-C(\phi)\right] \cot (\phi)$, for all $\phi \in(0, \pi / 2)$,

(iv) The volumetric flow rate in the $x$ direction due to the moving layer of the bed is described by the function

$$
q(x, t)=2 \int_{0}^{\frac{\pi}{2}} \tilde{q}(x, t, \phi) d \phi,
$$

where

$$
\tilde{q}(x, t, \phi)= \begin{cases}\lambda_{1} R h(x, t, \phi), & \text { for } h(x, t, \phi)<\Delta, \\ \lambda_{2}(a(x, t)) R \Delta, & \text { for } h(x, t, \phi) \geq \Delta,\end{cases}
$$

in such a way that $\tilde{q}(x, t, \phi) d \phi$ is just the volume of sediment passing through a section of width $d \phi$ per unit time consistently with the hypotheses $h \ll R$ and $\Delta \ll R$. The parameter $\lambda_{1}$ as well as the function $\lambda_{2}(a)$ have to be chosen so that $\tilde{q}(x, t, \phi)$ be continuous.

Obviously $C(\phi)$ takes its maximum at $\phi=\pi / 2$; therefore (4) is satisfied, for example, if $C^{\prime}(\phi) \leq\left[\frac{R}{a(x, t)}-C(\pi / 2)\right] \cot (\phi)$ provided that $a(x, t)<R / C(\pi / 2)$. Condition (4) can be easily interpreted: the z-coordinate of a point on the bed profile is given by

$$
z(\phi, x, t)=-(R-C(\phi) a(x, t)) \sin \phi .
$$

Provided that $a(x, t)$ is sufficiently small, condition (4) is equivalent to saying that $\partial z / \partial \phi<0$ for all $\phi \in[0, \pi / 2)$.

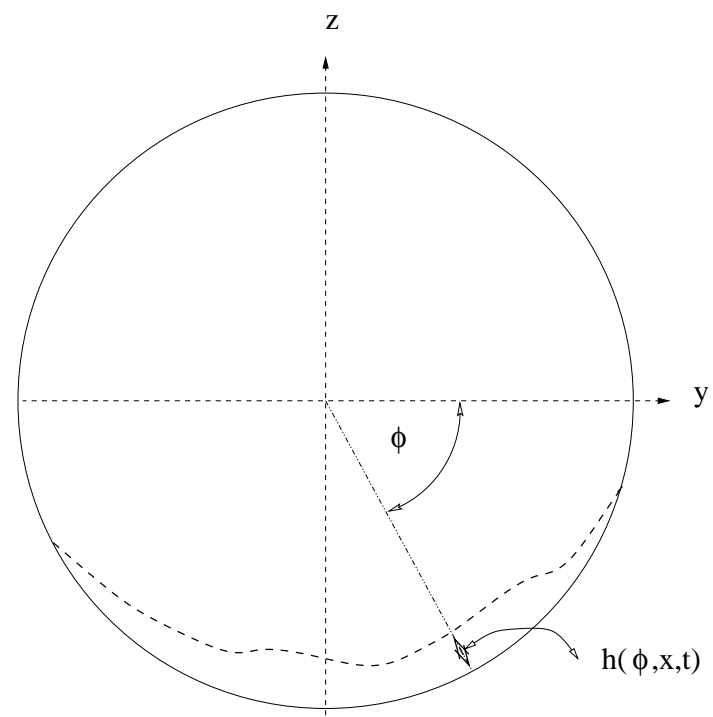

Figure 7: Function $h(\phi, x, t)$ describes the radial thickness of the bed; $\phi$ is the azimuthal coordinate.

Assumptions on $h$ are justified by the fact that in absence of experimental information it is convenient to choose working hypotheses which are both simple and meaningful. 
Let us define $g(2 \phi / \pi)=C(\phi) / C(\pi / 2)$ with $\phi \in[0, \pi / 2]$. Because of the hypotheses made on $C, g$ is invertible on $[0,1]$; let us also set $G(s)=\int_{0}^{s} g(u) d u$. The cross-sectional area $a(x, t)$ is described by $\iint_{\mathcal{A}} r d r d \phi$, where

$$
\mathcal{A}=\{(r, \phi) / \phi \in[0, \pi], \quad R-C(\phi) a(x, t) \leq r \leq R\} ;
$$

neglecting higher order terms (recall (ii)), this yields $C(\pi / 2)=1 /[\pi R G(1)]$. Moreover, for given $a(x, t)$, we have $0 \leq h(\phi, x, t) \leq C(\pi / 2) a(x, t)$; thus if $a \leq a_{o}:=\Delta / C(\pi / 2)$ then $h(\phi, x, t) \leq \Delta$ for all $\phi \in[0, \pi / 2]$. In this case, by recalling the definition of $q(x, t)$ in hypothesis (iv), we immediately obtain $q(x, t)=2 \lambda_{1} R \int_{0}^{\pi / 2} h(\phi, x, t) d \phi=$ $\lambda_{1} a(x, t)$, being $2 R \int_{0}^{\pi / 2} C(\phi) d \phi=1$. If, instead, $a(x, t)>a_{0}$, then there exists $\widehat{\phi}(a) \in[0, \pi / 2]$ such that $h(\widehat{\phi}, x, t)=\Delta$. Notice that $\widehat{\phi}(a)=(\pi / 2) g^{-1}\left(a / a_{0}\right)$, and that $\widehat{\phi}^{\prime}<0$. Therefore, for all cross-sections $x$ and instants $t$ for which $a(x, t)>a_{0}$, we have

$$
\begin{aligned}
q(x, t)=2 & {\left[\int_{0}^{\widehat{\phi}(a)} \lambda_{1} R h(\phi, x, t) d \phi+\int_{\widehat{\phi}(a)}^{\pi / 2} \lambda_{2}(a) R \Delta d \phi\right] } \\
& =\pi R \Delta\left[\lambda_{1} \frac{a}{a_{0}} G\left(\frac{2}{\pi} \widehat{\phi}(a)\right)+\lambda_{2}(a)\left(1-\frac{2}{\pi} \widehat{\phi}(a)\right)\right]
\end{aligned}
$$

Notice that $q\left(a_{0}^{+}\right)=q\left(a_{0}^{-}\right)$; indeed $\widehat{\phi}\left(a_{0}\right)=\pi / 2$ and

$$
q\left(a_{0}^{+}\right)=\pi R \Delta \lambda_{1} G(1)=(\pi R G(1) C(\pi / 2)) \lambda_{1} a_{0}=\lambda_{1} a_{0} .
$$

The simplest choice for $\lambda_{2}(a)$ is the linear one: $\lambda_{2}(a)=\lambda_{1}+\widehat{\lambda}_{1}\left(a-a_{0}\right)$, with $\widehat{\lambda}_{1}>0$.

The above analysis shows that we can write $q(x, t)$ as an explicit function of $a(x, t)$ : in particular we have

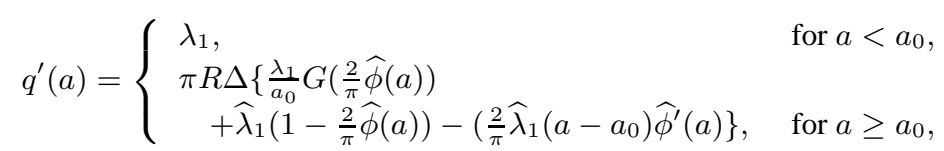

It is not difficult to check that $q^{\prime}(a)>0$; thus $q(a)$ is invertible in $\left[0, q_{\infty}\right)$ being $q_{\infty}=\lim _{a \rightarrow \infty} q$. If $\lim _{a \rightarrow a_{0}^{+}}(a-$ $\left.a_{0}\right) \frac{d}{d a} g^{-1}\left(a_{0} / a\right)=0$, then $q^{\prime}(a)$ is also continuous for all $a>0$. For future purpose it is also useful to notice that

$$
q^{\prime \prime}(a)= \begin{cases}0, & \text { for } a<a_{0}, \\ \pi R \Delta\left[\left(\frac{\lambda_{1}}{a}-2 \widehat{\lambda}_{1}\right) \widehat{\phi}^{\prime}(a)-\widehat{\lambda}_{1}\left(a-a_{0}\right) \widehat{\phi}^{\prime \prime}(a)\right], & \text { for } a \geq a_{0} .\end{cases}
$$

We are now ready to write down the dynamic equation for the evolution of the bed: a simple mass balance over a portion of the pipe of unit length yields

$$
\frac{\partial a}{\partial t}+q^{\prime}(a) \frac{\partial a}{\partial x}=\tilde{S}_{T}(x)
$$

where we set $\tilde{S}_{T}(x):=\frac{1}{\rho_{s}(1-\varepsilon)} S_{T}(x), \rho_{s}$ and $\varepsilon$ being the density of the settled material and the porosity of the bed respectively. The conversion factor $(1-\varepsilon)$ is needed since the rate of change of the cross-sectional area due to the settled material depends on its degree of porosity.

We complete equation (24) with the following initial-boundary conditions

$$
a(x, 0)=a(0, t)=0 .
$$

System (24)-(25) can be solved by the Method of Characteristics. Within the class of regular solutions, condition (25) imply that $a(x, t) \leq a_{0}$ in a neighbourhood $A=A_{1} \cup A_{2}$ of the initial lines $x=0$ and $t=0$. If

$$
\frac{1}{\lambda_{1}} \int_{0}^{\infty} \tilde{S}_{T}(u) d u<a_{0}
$$

then this neighbourhood covers the whole region $A=\{(x, t) / x \geq 0, \quad t \geq 0\}$, equation (24) is linear everywhere in $A$ and solution writes

$$
a(x, t)= \begin{cases}\frac{1}{\lambda_{1}} \int_{0}^{x} \tilde{S}_{T}(u) d u, & \text { for }(x, t) \in A_{1}:=\left\{(x, t) / 0<x<\lambda_{1} t\right\} \\ \frac{1}{\lambda_{1}} \int_{x-\lambda_{1} t}^{x} \tilde{S}_{T}(u) d u, & \text { for }(x, t) \in A_{2}:=\left\{(x, t) / 0<\lambda_{1} t<x\right\}\end{cases}
$$


Thus the solution is globally defined, grows below the line $t=\frac{1}{\lambda_{1}} x$, takes its maximum along $t=\frac{1}{\lambda_{1}} x$, and remains stationary for $t>\frac{1}{\lambda_{1}} x$. (see figure 8 ).
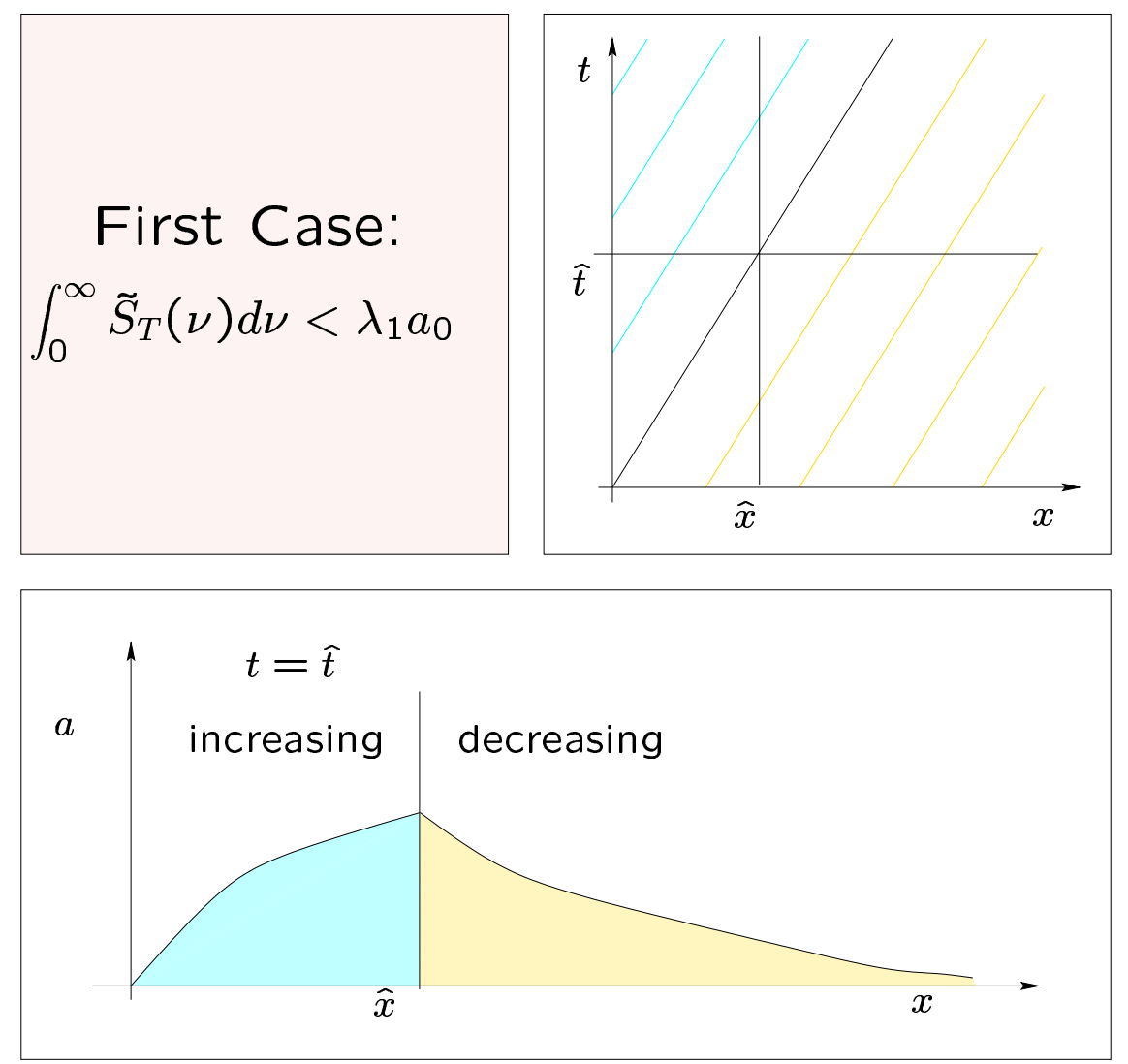

Figure 8: The region $A_{1} \cup A_{2}$ in the simplest case: the balance equation is linear and the characteristics are straight lines. At any instant $\widehat{t}, a(x, t)$ takes its maximum at $\widehat{x}=\lambda_{1} \widehat{t}$.

If (26) does not hold, the situation is much more complicated: besides regions $A_{1}$ and $A_{2}$ we have two further 
regions, $A_{3}$, and $A_{4}$ to consider. Indeed let us define $x_{0}>0$ such that

$$
\frac{1}{\lambda_{1}} \int_{0}^{x_{0}} \tilde{S}_{T}(u) d u=a_{0}
$$

We now have

$$
\begin{aligned}
& A_{1}=\left\{(x, t) / 0 \leq x \leq x_{0}, t \geq \frac{x}{\lambda_{1}}\right\}, \\
& A_{2}=\left\{(x, t) / 0 \leq x, 0 \leq t \leq t_{\gamma}(\xi), \xi \geq 0\right\},
\end{aligned}
$$

where the line $\gamma(\xi):=\left(x_{\gamma}(\xi), t_{\gamma}(\xi)\right)$ is defined by

$$
\left\{\begin{array}{l}
a_{0}=\frac{1}{\lambda_{1}} \int_{\xi}^{x_{\gamma}(\xi)} \tilde{S}_{T}(u) d u \\
t_{\gamma}(\xi)=\frac{1}{\lambda_{1}}\left(x_{\gamma}(\xi)-\xi\right) \\
\xi \geq 0, \quad x_{\gamma}(0)=x_{0}, \quad t_{\gamma}(0)=\frac{1}{\lambda_{1}} x_{0}
\end{array}\right.
$$

Region $A_{3}$ is covered by all characteristics coming out from the line $\left\{x=x_{0}, \quad t \geq \frac{x_{0}}{\lambda_{1}}\right\}$. These characteristics never intersect each other; the line

$$
t_{\sigma}(x)=\frac{x_{0}}{\lambda_{1}}+\int_{x_{0}}^{x} \frac{d u}{q^{\prime}\left(q^{-1}\left(\int_{0}^{\eta} \tilde{S}_{T}(u) d u\right)\right)}, \quad x \geq x_{0},
$$

bounds region $A_{3}$ from below. Region $A_{4}$ is finally defined as follows

$$
A_{4}:=\left\{(x, t) / x \geq x_{0}, \quad t_{\gamma}(\xi) \leq t \leq t_{\sigma}(x), \quad \xi \geq 0\right\}
$$

In region $A_{1} \cup A_{2}$ system(24) is still linear and solution writes as before. In region $A_{3} \cup A_{4}$ system(24) is fully nonlinear. In $A_{3}$ solution is stationary (as in $A_{1}$ ) and writes

$$
a(x)=q^{-1}\left(\int_{0}^{x} \tilde{S}_{T}(u) d u\right)
$$

being $\frac{\partial a}{\partial x}>0, a(x, t)$ is increasing in $A_{1} \cup A_{3}$. In region $A_{4}$ the analysis is more complicated since characteristics may intersect each other. (see figure 9). Global existence of a solution in this case is guaranteed only if $q^{\prime \prime}(a)<0$ and $q_{\infty}>\int_{0}^{\infty} \widetilde{S}_{T}(u) d u$. If $q^{\prime \prime}(a)>0$ the existence is guaranteed only for $t<\hat{t}$, where $\hat{t}$ can be explicitly determined as a function of $\tilde{S}_{T}$ and $\Delta$ (see [28] for details).

For $q_{\infty} \leq \int_{0}^{\infty} \widetilde{S}_{T}(u) d u$ the above conclusion continues to be true but the definition of $q(a)$ needs to be slightly modified: indeed the model is physically significant only as long as $a(x, t)$ remains small. Therefore we can always think of $q(a)$ as defined by (20) until $a$ exceeds some upper bound and then extend $q(a)$ (for example linearly) in order to get $q_{\infty}=+\infty$. After that, condition $q_{\infty}>\int_{0}^{\infty} \widetilde{S}_{T}(u) d u$ can be considered always satisfied.

Actually $q^{\prime \prime}(a)>0$ means that the transport mechanism is rather efficient: this could lead to the formation of a shock front along the bed profile. Thus a classical solution does no longer exist and weak solutions should be considered, although it seems unrealistic that the moving bed can really exhibit any jump, owing to its incoherence. In region $A_{4}$ we have $\frac{\partial a}{\partial x}<0$; moreover $\frac{\partial a}{\partial x}$ is continuous except along the curve $t=t_{\sigma}(x)$ where $a(x, t)$ takes its maximum for each $t$. For a given $\bar{t}>0$, let $\bar{x}(\bar{t})$ be defined by $t_{\sigma}(\bar{x}(\bar{t}))=\bar{t}$. Then we can write

$$
\max _{t<\bar{t}} a(x, t)=a(\bar{x}(\bar{t}), \bar{t})
$$

and prove that the estimate

$$
a(x, t) \leq q^{-1}\left(\int_{0}^{\infty} \widetilde{S}_{T}(u) d u\right)
$$

holds everywhere in the existence domain of $a(x, t)$. 

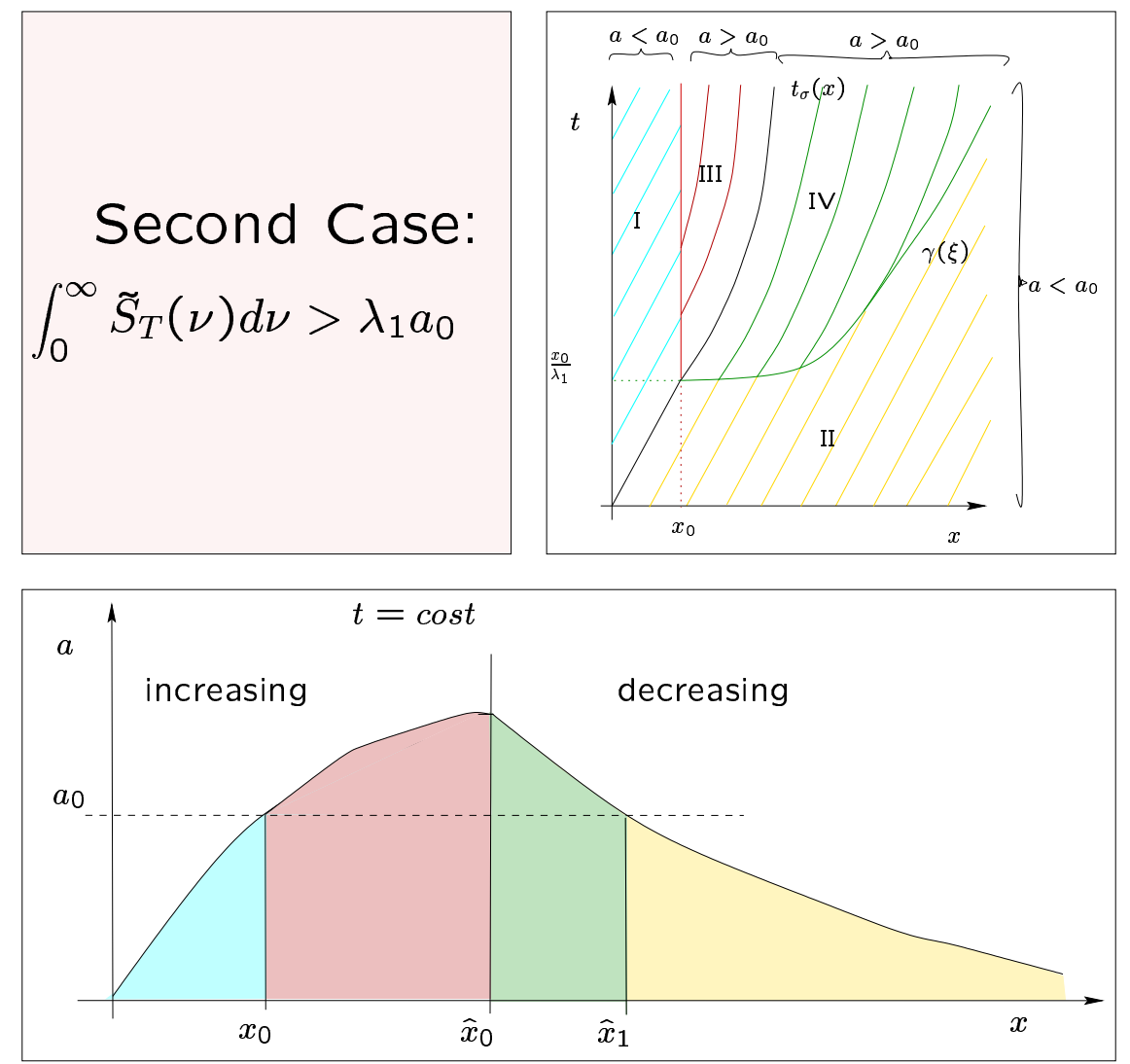

Figure 9: The region $A_{1} \cup A_{2} \cup A_{3} \cup A_{4}$ when the balance equation is nonlinear. In this case the characteristic lines may intersect in region $A_{4}$. As long as $t \leq x_{0} / \lambda_{1}$ the picture is the same as in figure 8 . For $t>x_{0} / \lambda_{1}, a(x, t)$ takes its maximum along $t_{\sigma}(x)$; at $x_{1}, a(x, t)$ decreases below the value $a_{0}$ again

\section{Numerical simulations}

The function $g(\psi)$ assigns a pre-defined geometry to the cross-sectional profile of the bed. We choose $g(u)=u(2-u)$ but more general choices are possible such as $g(u)=u^{k}(2-u)^{k}$ : for $k>1$ the bed shows thinner edges (see figure 10). 


\section{Cross Sectional Profiles}
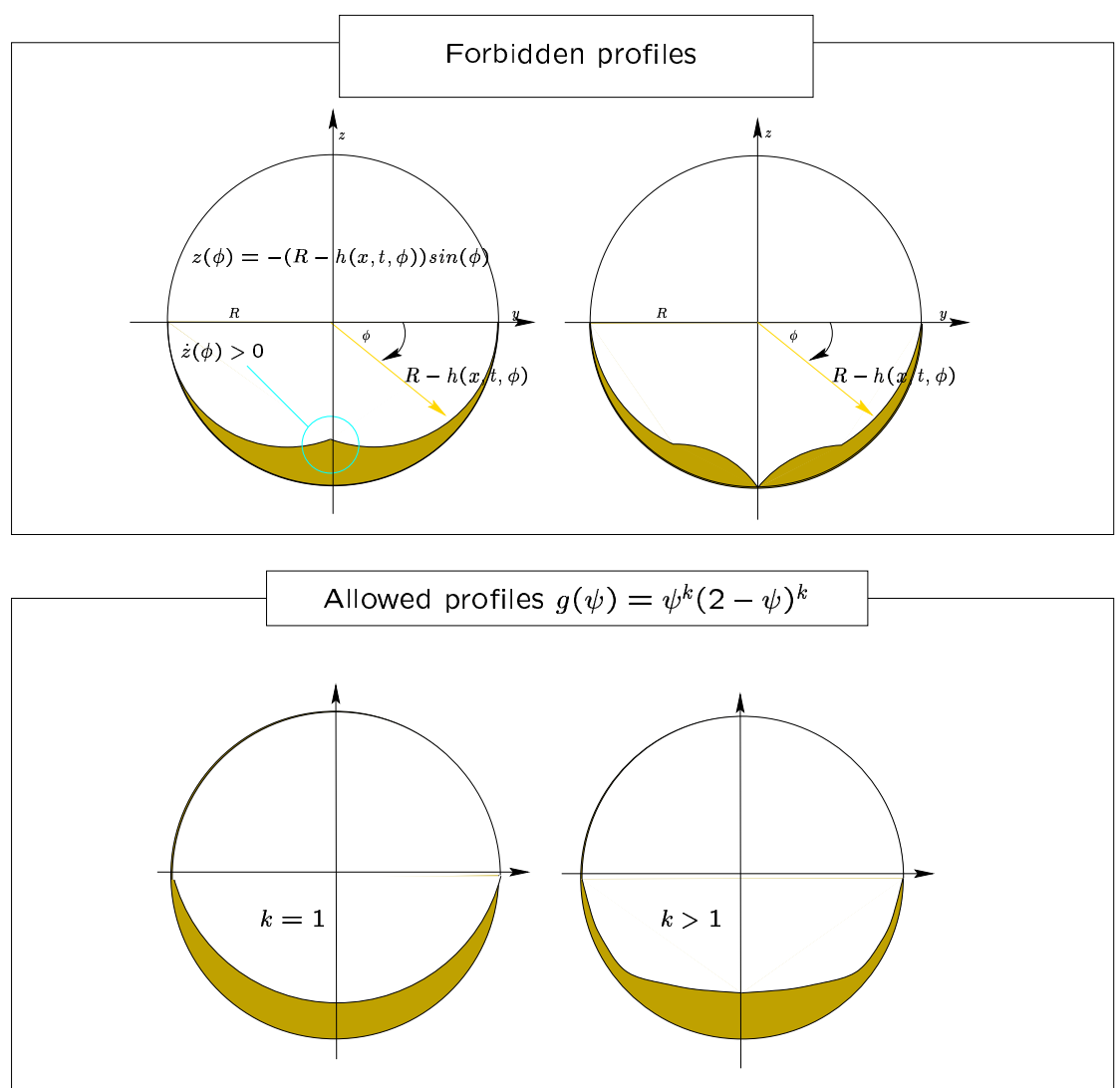

Figure 10: Axdmissible and non-admissible bed profiles: the forbidden profiles violate the hypotheses (iii,4) we made on function $C(\phi)$.

For $k=1$ we get $\widehat{\phi}(a)=\frac{\pi}{2}\left(1-\sqrt{1-\frac{a_{0}}{a}}\right)$, and so

$$
q^{\prime \prime}(a)=\omega(\phi):=\frac{\pi R^{2} a_{0}\left(3 \hat{\lambda}_{1} a_{0}-2 \lambda_{1}\right)}{8 a^{3} \sqrt{1-\frac{a_{0}}{a}}} ;
$$

moreover condition (iii,4) reads

$$
a(x, t) \leq \frac{\pi^{3} R^{2}}{6(\pi-2 \phi) \tan \phi+\phi(\pi-\phi)},
$$


which is satisfied for any $\phi \in[0, \pi]$ if $a(x, t) \leq \min _{\phi \in[0, \pi]} \omega(\phi)=\frac{2 \pi^{2}}{24+3 \pi^{2}} \pi R^{2}$.

The parameter $\lambda_{1}$ has the physical meaning of a "velocity", namely the mean velocity of the dynamic layer of sediment. We decided to set $\lambda_{1}=V_{x}\left(R-\frac{\Delta}{2}\right)$ (though other choices are possible) so identifying the velocity of the moving layer with that of the main flow of CWS at $\frac{\Delta}{2}$ units far from the bottom pipe wall.

If we choose $\widehat{\lambda}_{1}=0$, then $\lambda_{2}(a)=\lambda_{1}$ and (20) yields a finite value of $q_{\infty}$; thus we need to modify the definition of $q(a)$ as explained before in order to avoid critical conditions in the existence proof. Because of $(31), \widehat{\lambda}_{1}=0$ implies $q^{\prime \prime}<0$ and the solution is globally defined.

In the case $\widehat{\lambda}_{1} \neq 0$, the dynamic layer moves with mean velocity $\lambda_{1}$ for $h<\Delta$ and grows linearly as $a-a_{0}$ for $h>\Delta$. This case is more complicated because, as we said before, the existence of a classical solution cannot be guaranteed for all times. All simulations to be shown next have been carried out (for simplicity) for $\widehat{\lambda}_{1}=0$. Therefore, from now on, we definitely assume $\lambda_{2}(a)=\lambda_{1}$.

The analysis developed in Section 4 shows that the value of the quantity $S_{\infty}=\int_{0}^{\infty} \widetilde{S}_{T}(u) d u$ (which we assume to be finite, consistently with the real situation) plays a central role in discriminating two distinct situations. If $S_{\infty}<\lambda_{1} a_{0}$ then the bed never reaches the level $\Delta$; therefore it remains fully dynamic never developing a static sub-layer. If, on the contrary, $S_{\infty}>\lambda_{1} a_{0}$ a static layer definitely develops which keeps growing below the dynamic one as shown in figure 6 .

Given a point at distance $x$ from the origin, $a(x, t)$ reaches its maximum value $a_{\max }(x)$ at that point, at time $t_{\sigma}(x)$; it turns out that

$$
a_{\max }(x)=a\left(x, t_{\sigma}(x)\right)=q^{-1}\left(\int_{0}^{x} \widetilde{S}_{T}(u) d u\right) .
$$

Once $a(x, t)$ has reached its maximum value at a given point, the graph of $a$ for $\hat{x}<x$ remains stationary, i.e. independent of $t$, for all $t \geq t_{\sigma}(x)$; in other words the bed "crest" moves ahead towards infinity just like a wave, leaving a stationary "frozen" profile behind.

The choice of $\Delta$ is a very delicate matter. This is truly the key parameter of the model (as it is quite evident from the above choice for $\lambda_{1}$ ). On the other hand direct experimental indications about $\Delta$ seem actually inaccessible for several reasons. First of all $\Delta$ is essentially a dynamical parameter, i. e. it cannot be measured at rest. Secondly, accurate dynamical measurements are not actually available even with sophisticated instruments. Last but not least CWS is an opaque substance and direct optical measurements are out of discussion.

For all these reasons we were forced to identify $\Delta$ indirectly as follows: we focussed upon the richest set of experimental data available, namely those obtained at the flow rate $Q^{\star}=250 \mathrm{~m}^{3} / \mathrm{h}^{-1}$. Once these are fixed as "reference"data, we select $\Delta$ in such a way that numerical simulation based on our model, have to fit the experimentally observed behaviour. Then we assume that $\Delta$ depends linearly on $Q$ with $\Delta(0)=0$. This approach led us to set $\Delta=1.8 \frac{Q}{Q^{\star}} \mathrm{cm}$ and so to the following values

$$
\begin{array}{l|cccc}
\mathrm{Q}\left(\mathrm{m}^{3} / h\right) & 100 & 150 & 250 & 450 \\
\hline \Delta(\mathrm{cm}) & 0.7 & 1.0 & 1.8 & 3.2 \\
& \text { Table } 1 & &
\end{array}
$$

for the other flow rates used in our simulations.

Let us now define $t_{c r}$ as the time instant at which the thickness $h$ of the bed reaches a given critical level $h_{c r}$. We know that $t=t_{\sigma}(x)$ is the steady curve "drawn"by the maxima of $a(x, t)$ as long as time elapses and the bed keeps growing. If $\left(x, t_{\sigma}(x)\right)$ is a point on this curve in the $(x, t)$-plane then, bearing in mind (i) in Section 4, (32), and the fact that $t_{\sigma}(x)$ is invertible (see [28]), we get

$$
h_{c r}=q^{-1}\left(\int_{0}^{t_{\sigma}^{-1}\left(t_{c r}\right)} \widetilde{S}_{T}(u) d u\right) \max _{\phi \in[0, \pi / 2]} C(\phi) .
$$

Let us come now to numerical simulations. The rheological parameters of CWS are just those already mentioned at the end of Section 2. The size distribution of the settling material is described in [15] and [18].

The pipe radius $R$ and length $L$ are set equal to $25 \mathrm{~cm}$ and $100 \mathrm{~km}$ respectively. Figure 11 shows the curves $\widetilde{S}_{T}(x)$ at all flow rates we considered. 


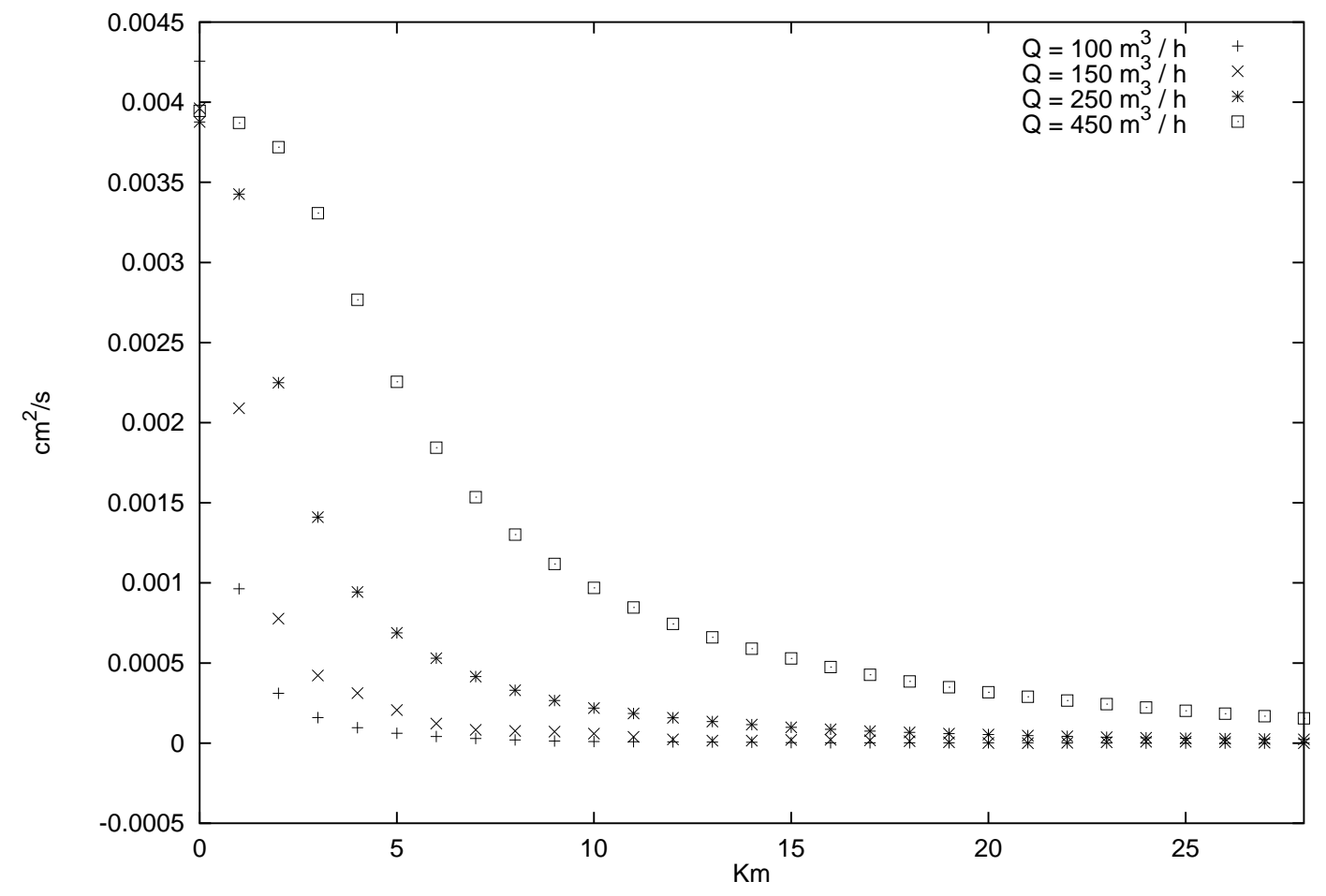

Figure 11: Function $S_{T}(x)$ at various flow rates.

Table 2 shows critical times and distances needed by the static sediment to reach $2 \%(=1 \mathrm{~cm})$ of the pipe diameter.

\begin{tabular}{ccccc}
$Q\left(\mathrm{~m}^{3} / \mathrm{h}\right)$ & $\Delta(\mathrm{cm})$ & $\mathrm{Gap}(\mathrm{cm})$ & $t_{c r}$ (days) & $x_{c r}(\mathrm{Km})$ \\
\hline \multirow{2}{*}{100} & 0.7 & 3.5 & 0.4 & 0.48 \\
150 & 1.0 & 4.2 & 0.5 & 1.24 \\
250 & 1.8 & 5.4 & $\infty$ & $\geq 100$ \\
450 & 3.2 & 7.1 & $\infty$ & $\geq 100$
\end{tabular}

For $Q=250$ and $=450 \mathrm{~m}^{3} / \mathrm{h}, t_{c r}$ and $x_{c r}$ are out of the computational range. Next table shows, just for these flow rates, both time and distances for which the dynamic layer rises up to $1.5 \mathrm{~cm}$ (no static layer develops in these cases).

\begin{tabular}{cccc}
$Q\left(\mathrm{~m}^{3} / \mathrm{h}\right)$ & $\Delta(\mathrm{cm})$ & $t($ days $)$ & $x(\mathrm{Km})$ \\
\hline 250 & 1.8 & 0.39 & 4.3 \\
450 & 3.2 & 0.38 & 10.1 \\
\multicolumn{4}{c}{ Table 3}
\end{tabular}

However $100 \mathrm{~m}^{3} / \mathrm{h}$ is generally considered a very low regime (for $R=25 \mathrm{~cm}$ ) from the point of view of economical convenience, since industrial pumps actually allow much higher regimes. For high values of $Q$ the gap between the rigid core and the pipe wall is large enough in order $h_{c r}=2 R / 100$ continue to be a very significant parameter. A more interesting choice might be the time $T_{c r}$ needed by the whole system to reach a state in which the thickness of the static layer is above $h_{c r}=2 R / 100$ over a longitudinal section of the pipe with length $L_{c r} \simeq L / 100(=1 \mathrm{Km})(L=$ total length of the pipeline). From the industrial point of view (at flow rates $\geq 250 \mathrm{~m}^{3} / \mathrm{h}$ ) this may be just the time after which a pipe shut-down and cleaning for sediment removal is highly recommended. Table 4 shows $t_{c r}, x_{c r}$, and $T_{c r}$ for $Q=250 \mathrm{~m}^{3} / \mathrm{h}$ and $L=100 \mathrm{Km}$ for various values of $\Delta$. It was just this analysis to suggest the value $1.8 \mathrm{~cm}$ as the one for which our simulations fit better available data. 


\begin{tabular}{cccc}
$\Delta(\mathrm{cm})$ & $t_{c r}($ days $)$ & $x_{c r}(\mathrm{Km})$ & $T_{\text {cr }}($ days $)$ \\
\hline 1.4 & 0.71 & 3.57 & 1.01 \\
1.5 & 0.97 & 4.99 & 1.35 \\
1.6 & 1.51 & 7.5 & 2.08 \\
1.7 & 3.20 & 14.2 & 3.85 \\
1.8 & $\infty$ & $\infty$ & $\infty$ \\
1.9 & $\infty$ & $\infty$ & $\infty$ \\
2.0 & $\infty$ & $\infty$ & $\infty$
\end{tabular}

Figures 12, 13, 14 below show the static and dynamic bed profiles for $Q=250 \mathrm{~m}^{3} / \mathrm{h}$ and $\Delta=1.8 \mathrm{~cm}$ when $t=1$ day, $t=5$ days, and $t=10$ days. As it can be seen, the static sediment is still below the critical level of $1 \mathrm{~cm}$ even after 10 days.

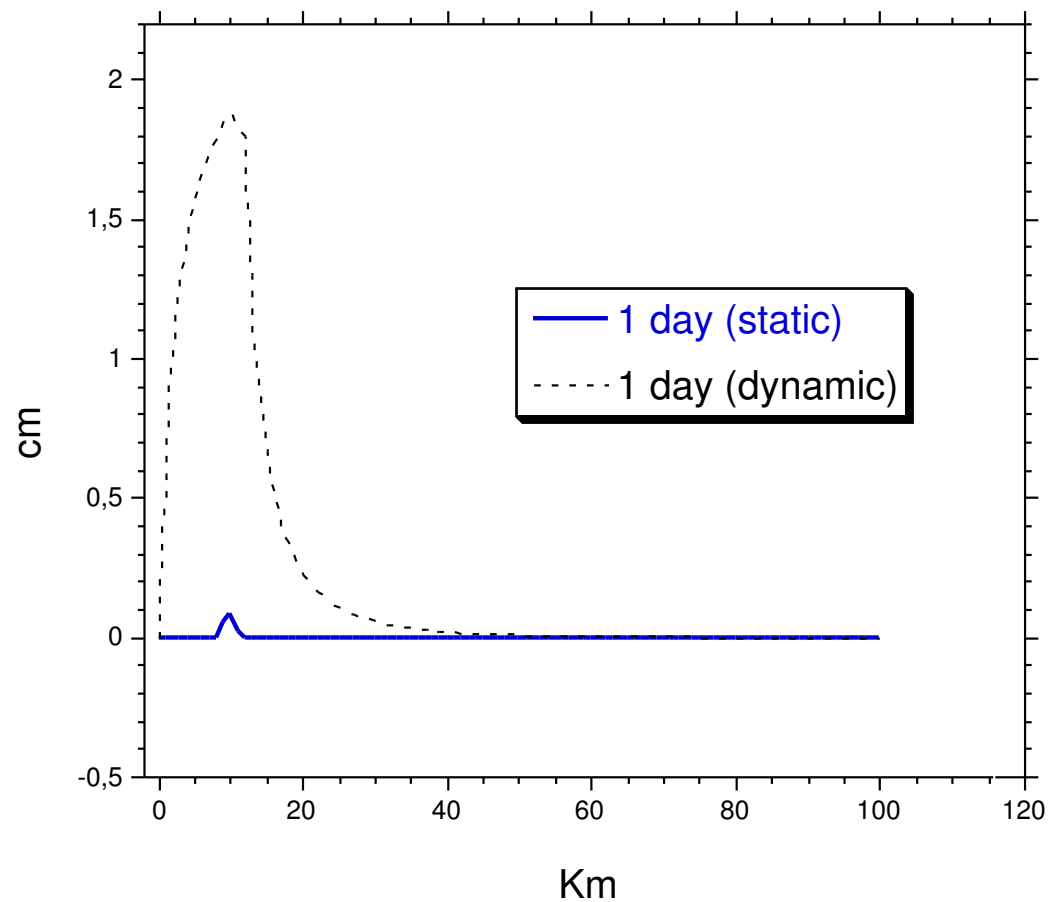

Figure 12: Static and mobile sediment profile after one day at $250 \mathrm{~m}^{3} / \mathrm{h}$ 


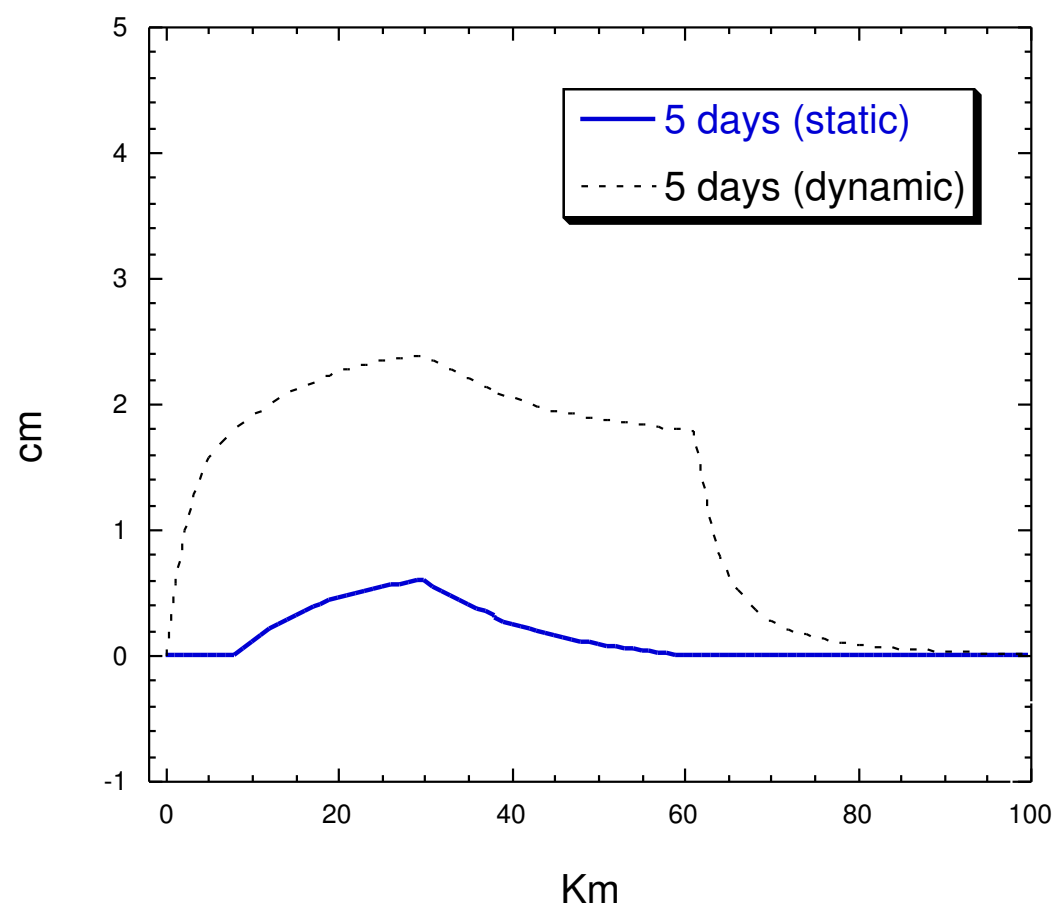

Figure 13: Static and mobile sediment profile after five days at $250 \mathrm{~m}^{3} / \mathrm{h}$ 


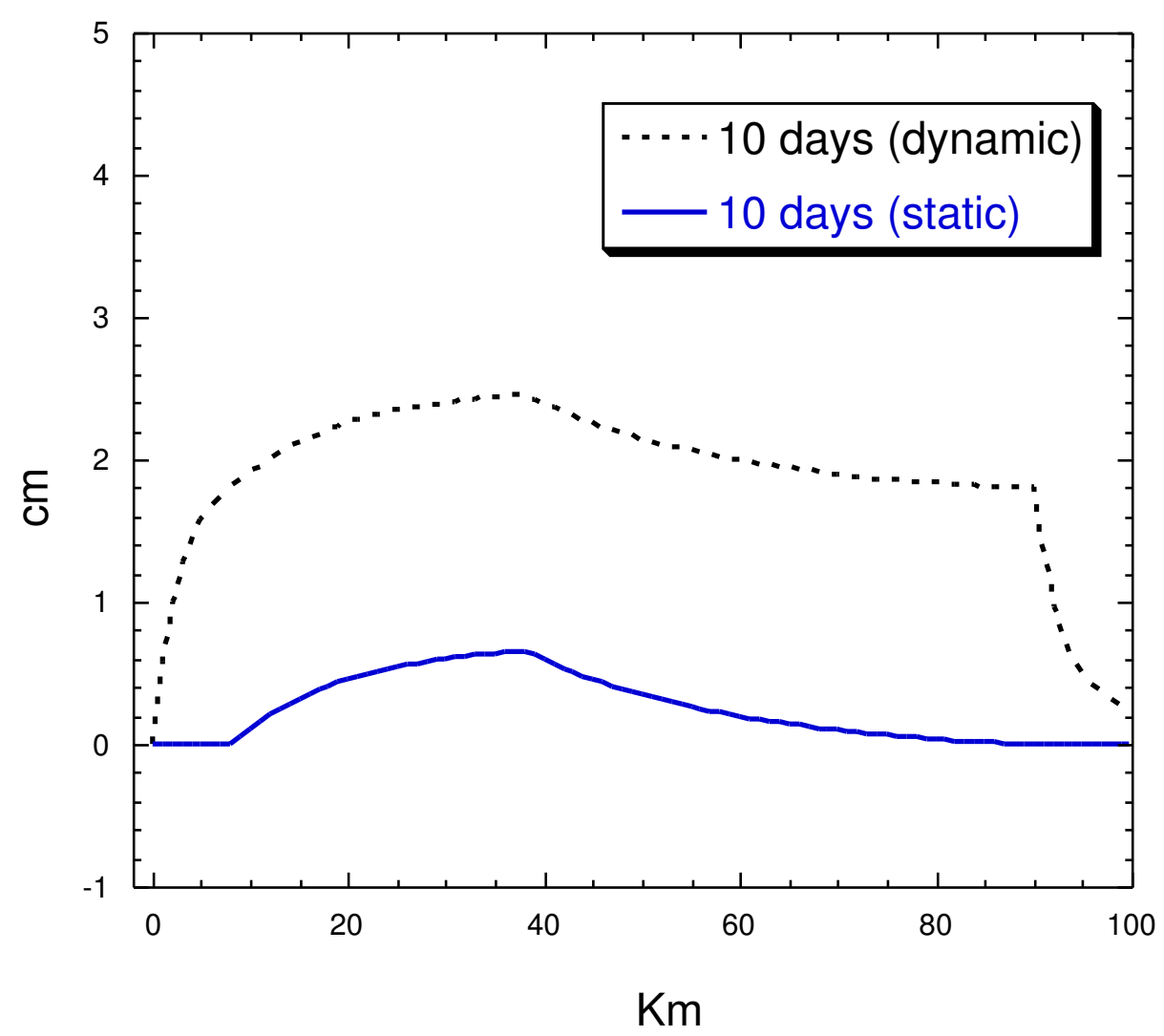

Figure 14: Static and mobile sediment profile after ten days at $250 \mathrm{~m}^{3} / \mathrm{h}$

\section{Conclusions and open problems}

In this review article we tried to report some of the results obtained in modelling the problem of particle sedimentation in a sheared slurry. Among other things, we developed a functional method to determine $v_{s}$ (described in the Appendix). However this approach has been applied only to the simple Newtonian case. For a Bingham fluid this method appears rather complicated and has not been developed yet. This forced us to follow the more traditional approach based on the assumption that $v_{s}$ has some pre-assigned dependence on the radius of the particle.

As far as we know the model for the evolution of the bed is totally new and, despite its simplicity, seems capable to predict the critical values to operate an industrial pipeline under safe conditions. The numerical code developed by A. Mancini is property of Snamprogetti.

While for the purposes of industrial designers the assumptions we made are rather reasonable and make the model easy to handle, there still are some side questions which would deserve further investigations. We list some of them.

The model presented here does not consider the sliding motion of particles once they have touched the wall. This would add a better information about the cross sectional profile of the bed which in the model is assigned a priori. This is certainly a very difficult problem also because very few experimental information is available.

The existence proof for equation (24) guarantees global existence in time if $q^{\prime \prime}<0$. If this condition is not fulfilled, a shock develops and it seems more reasonable to switch to a different transport model. This analysis is not expected to be very hard but is still missing. 


\section{Appendix: A functional approach to settling velocity of particles in a finite container}

We considered a liquid container $V$ that can be either a cylinder or a rotational viscometer. The container is filled up with a liquid $\mathcal{L}$ whose constitutive law we do not need to specify at present. Therefore $V$ is characterized by its height $l$ and by the radii $R_{1} \geq 0$ and $R_{2}>R_{1}$ of the inner and outer cylinder respectively. Indeed our approach bypasses not only the rheological nature of $\mathcal{L}$ but also a detailed description of some intricate aspects of sedimentation such as fluid-particle and/or particle-particle interactions, wall-effects, particle shape effects, etc... (see [12],[15],[34]). Let us suppose that a population $\mathcal{P}$ of particles is uniformly distributed in $\mathcal{L}$ at time $t=0$. If the particle density $\rho_{\mathcal{P}}$ is greater than the liquid density $\rho_{\mathcal{L}}, \mathcal{P}$ is expected to settle toward the bottom of $V$. If $\mathcal{L}$ has a yield stress there may be a fraction of $\mathcal{P}$ which never settles, although the unsettled fraction generally changes according to the shearing conditions.

The family $\mathcal{P}$ is supposed to be represented by a rather large number $\mathcal{N}$ (say $\geq 10^{5}$ ) of particles with random shape and very small size.

Let us point our attention over a cross-sectional cell $C$ of the container $V$ of thickness $h$ with the top and the bottom bases placed respectively at $z-h(z>h)$ and $z \geq 0$, the $z$-axis being directed as the gravity vector $(z=0$ denotes the top of $V$ ). The quantity to be measured is the net mass-variation in $C$ due to the settling of $\mathcal{P}$.

As we already said in section 2, the stationary settling regime is generally reached almost instantaneously and the observation cell $C$ will experience an emptying wave starting from the moment in which the heaviest particle which left $z=0$ at $t=0$ has reached the level $z-h$.

Let us recall equation (10): for given $\dot{\gamma}, \mathcal{M}$ measures the mass due to $\mathcal{P}$ present in $C$ at time $t$.

It is more convenient to write (10) as follows

$$
\mathcal{M}^{\star}(v ; t, \dot{\gamma})=\lambda \int_{U} \Omega(\delta) \Phi[v(\delta) ; t] d \delta,
$$

where

$$
\mathcal{M}^{\star}=1-\mathcal{M} / \mathcal{M}_{o}, \quad \lambda=-\rho_{\mathcal{P}} \Sigma K / \mathcal{M}_{o}, \quad U=\operatorname{supp} \Omega \cap\left[\delta_{o}(\dot{\gamma}), \infty\right),
$$

and $\delta_{o}$ is a possible critical radius defined by (8).

For $\{z, h, \dot{\gamma}\}$ given ${ }^{6}$, the right hand side of (33) defines a (formal) nonlinear operator $N$ which maps $v(\delta)$ into the function

$$
N v(t)=\lambda \int_{U} \Omega(\delta) \Phi[v(\delta) ; t] d \delta .
$$

The function $1-\mathcal{M}^{\star}(t)$ measures the percentage of $\mathcal{P}$ present in $C$ at time $t$. Since $\mathcal{M}^{\star}(t)$ is a measurable data, the functional equation

$$
N v=\mathcal{M}^{\star}
$$

can be used, in principle, to determine $v$.

As emphasized in [34] the fully nonlinear problem is rather complicated. In this case it is possible and reasonable, on the basis of a suitable physical approximation, to linearize the problem. This leads to the functional equation

$$
A w=g,
$$

where $A$ is a linear operator. In particular, (36) turns out to be a Fredholm equation of the first kind, which, as it is well known, is ill-posed in the sense of Hadamard.

The choice of the solution (which will be called "reference" solution) nearby which to linearize (35) depends mainly on the physical situation. For example if the fluid $\mathcal{L}$ is almost Newtonian it may be reasonable to linearize (35) around (2), while for a Bingham plastic other choices are more suitable.

Let us decompose the unknown $u$ into the "reference" solution $v$ plus a "perturbation" $w$ due to all effects mentioned at the beginning of Section 2. Then

$$
N u \approx\left(N^{\prime}[v], w\right)+N v,
$$

where $N^{\prime}[v]$ is the Frechét derivative of $N$ calculated in $v$ and (.,.) is a scalar product to be specified. To calculate $N^{\prime}[v]$ explicitly we shall make some assumptions on the class of reference solutions $v$. It is quite natural to ask that

\footnotetext{
${ }^{6}$ From the experimental point of view this means to have definitely fixed $C$ and the shearing condition for $\mathcal{L}$.
} 
$v>0$ in $\left(\delta_{o},+\infty\right), v=0$ in $\left[0, \delta_{o}\right]$, and that $d v / d \delta>0$ in $\left(\delta_{o},+\infty\right)$. Here $\delta_{o} \geq 0$ as a function of $\dot{\gamma}$ is supposed to be known. Consequently there exists the inverse $\tilde{v}$ of $v$ defined over $[0,+\infty)$ and we can write

$$
\Phi= \begin{cases}0, & \delta \leq \widetilde{v}\left(c_{1}(t)\right) \\ z-h-v(\delta) t, & \widetilde{v}\left(c_{1}(t)\right) \leq \delta \leq \widetilde{v}\left(c_{2}(t)\right), \\ -h, & \delta \geq \widetilde{v}\left(c_{2}(t)\right)\end{cases}
$$

where $c_{1}(t)=\frac{z-h}{t}$ and $c_{2}(t)=\frac{z}{t}$. Then an elementary calculation shows that

$$
\left(N^{\prime}[v], w\right)=-\lambda \int_{A} t \Omega(\delta) \mathcal{X}_{I(t)}(\delta) w(\delta) d \delta,
$$

where $\mathcal{X}_{I(t)}(\delta)$ is the characteristic function of $I(t)=\left[\delta_{1}(t), \delta_{2}(t)\right]$ and $\delta_{i}(t)=\widetilde{v}\left(c_{i}(t)\right)$. The formal structure of our problem is thus the following

$$
A w:=\left(N^{\prime}[v], w\right)=\mathcal{M}^{\star}-\lambda N v .
$$

In other words, the right hand side of (39) denotes the difference between the effective emptying wave $\mathcal{S}$ (observable) and a reference emptying wave corresponding to $v$. If $v$ is assigned, equation (40) is the explicit form of equation (36) in the unknown $w$ we were looking for. However in a typical experiment $\mathcal{S}$ is available only over a finite set of time values $t_{i=1, \ldots, n}$. Thus $\mathbf{g}:=\mathcal{M}^{\star}-\lambda N v$ is a vector in $R^{n}$ rather than a function of $t$ and so equation (40) is actually a Fredholm equation of first kind with discrete data. It is quite natural therefore to discretize also the kernel $k(\delta, t):=\lambda t \Omega(\delta) \mathcal{X}_{I(t)}(\delta) w(\delta)$ by putting $k_{i}(\delta):=k\left(\delta, t_{i}\right)$, the operator $A$ by putting $A_{i} w:=\int_{U} k_{i}(\delta) w(\delta) d \delta$, and to consider just the discretized problem

$$
A_{i} w=\mathbf{g}_{i}, \quad(i=1, \ldots, n) .
$$

It is easy to see that each $A_{i}$ is bounded in $L^{2}(U)$ being

$$
\left|A_{i} w_{2}-A_{i} w_{1}\right| \leq C_{i}|| w_{2}-w_{1} \|
$$

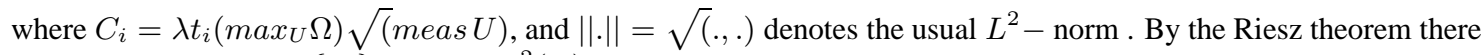
exists a set of functions $\left\{\phi_{i}\right\}_{i=, \ldots, n}$ in $L^{2}(U)$ such that

$$
A_{i} w=\left(w, \phi_{i}\right), i=1, \ldots, n .
$$

The precise formulation of the problem is thus the following:

given the set $\left\{\phi_{i}\right\}_{i=1, \ldots, n}$ in $L^{2}(U)$ and the data $\mathbf{g} \in R^{n}$, find a function $w \in L^{2}(U)$ such that

$$
\left(\phi_{i}, w\right)=\mathbf{g}_{i}, i=1, \ldots, n .
$$

Problems like these have been deeply investigated by Bertero et al. in [7],[8], where the concept of solution is generalized in such a way to overcome the following intrinsic difficulty of (44): this equation provides information only about a finite number of components of $w$ which in turn implies a lack of uniqueness and, if $n$ is large, also that of numerical stability. These unpleasant features have to be related to the ill-posedness of the original infinite-dimensional problem (40) as it is known from the theory of Fredholm equations of first kind.

To proceed further let us denote by $\mathcal{A}: w \in L^{2}(U) \rightarrow \mathbf{g} \in R^{n}$ the mapping defined according to the rule

$$
(\mathcal{A} w)_{i}=\left(w, \phi_{i}\right), i=1, \ldots, n,
$$

where $(\mathcal{A} w)_{i}$ denotes the $\mathrm{i}$-th component of $\mathcal{A} w$; of course $(\mathcal{A} w)_{i}=A_{i} w$. The mapping $\mathcal{A}$ is onto when the $\phi_{i}$ are linearly independent (a circumstance which is hard -if not impossible- to check in practice); otherwise the range of $\mathcal{A}$ has dimension $n^{\prime}<n$. Let us denote by $X$ the subspace of $L^{2}(U)$ spanned by the $\phi_{i}$. Regardless of the $\phi_{i}$ being independent or not, the orthogonal complement $X_{\perp}$ of $X$ is just the null space $\mathcal{N}(\mathcal{A})$ of $\mathcal{A}$. The adjoint $\mathcal{A}^{*}$ of $\mathcal{A}$, defined by $(\mathcal{A} w, \mathbf{g})_{R^{n}}=\left(w, \mathcal{A}^{*} \mathbf{g}\right)_{L^{2}(U)}$ transforms $\mathbf{g}$ into an element of $X$ since if $w \in X_{\perp}$ then $\left(w, \mathcal{A}^{*} \mathbf{g}\right)_{L^{2}(U)}=0$. Evidently $\mathcal{A}^{*} \mathbf{g}=\sum_{i=1}^{n} g_{i} \phi_{i}$. Therefore it is clear that, given $\mathbf{g} \in R^{n}$, the problem of finding a $w \in L^{2}(U)$ such that

$$
\mathcal{A} w=\mathbf{g},
$$

when the $\phi_{i}$ are not linearly independent, has a solution if and only if $\mathbf{g} \in X$. If $\mathbf{g} \notin X$ one defines a pseudo-solution of equation (46) as any $\widetilde{w} \in X$ such that

$$
\|\mathcal{A} \widetilde{w}-\mathbf{g}\|_{R^{n}}=\text { minimum }
$$


This definition is well-motivated from the physical point of view: the minimum (47) is just the norm of the component of $\mathbf{g}$ orthogonal to $R^{n^{\prime}}$ and this component is purely an effect of the experimental errors (see [7]). Since the set of pseudo - solutions is closed and convex there exists a unique pseudo-solution of minimal norm called generalized solution and denoted by $w^{\dagger}$. This solution always depends continuously upon the data.

To obtain an explicit representation of $w^{\dagger} \in X$ we need to construct the set $\left\{\lambda_{i}, \epsilon_{i}, \mathbf{e}_{i}\right\}$ (called the singular system of $\mathcal{A}$ ) by solving the problem $\mathcal{A} \epsilon_{i}=\lambda_{i} \mathbf{e}_{i}, \mathcal{A}^{*} \mathbf{e}_{i}=\lambda_{i} \epsilon_{i}$. This set can be computed with standard techniques (see [7] for more details); in particular it turns out that the numbers $\lambda_{i}^{2}$ are the eigenvalues of the Gram matrix associated with the functions $\phi_{i}$, that are all positive, and that their number is equal to the number $n^{\prime} \leq n$ of linearly independent $\phi_{i}$. This bearing in mind, the representation formula for $w^{\dagger}$ is the following:

$$
w^{\dagger}=\sum_{i=1}^{n} \frac{1}{\lambda_{i}}\left(\mathbf{g}, \mathbf{e}_{i}\right) \epsilon_{i} .
$$

Clearly, if there are only $n^{\prime}<n$ linearly independent $\phi_{i}$, we have only $n^{\prime}$ terms in equation (48).

The maximum eigenvalue $\lambda_{M}$ and the minimum eigenvalue $\lambda_{m}$ have an important meaning: the ratio $c(\mathcal{A})=$ $\lambda_{M} / \lambda_{m}$ is called the "condition number" of $\mathcal{A}$. If $c(\mathcal{A}) \gg 1$, the problem of computing $w^{\dagger}$, although well-posed, is "ill-conditioned". This circumstance is due to the ill-posedness of the related infinite-dimensional problem and could lead to large numerical errors. The situation does not improve by increasing the number $n$ of available data $g_{i}$, since in this case the finite-dimensional problem becomes "closer" to the infinite-dimensional one.

The above method was applied to a population of sand particles uniformly dispersed in glycerine ${ }^{7}$ : sand is $12 \%$ by weight and $9 \%$ by volume, with density of $2.67 \mathrm{~g} / \mathrm{cm}^{3}$ and "diameter" distribution between 100 and $150 \mu \mathrm{m}$. The container is just a static cylinder with diameter $2.64 \mathrm{~cm}$ and height of $14 \mathrm{~cm}$ held at a temperature of $4 C^{0}$. At this temperature the glycerine viscosity is $\approx 6.13 \mathrm{~Pa} \times s$ and the calculated coefficient $\frac{2 g\left(\rho_{s}-\rho_{l}\right)}{9 \eta}$ in (2) turns out to be $\approx 5.0 \mathrm{~cm}^{-1} \times s^{-1}$. The chosen observation cell lies between $z=4.5 \mathrm{~cm}$ and $z+h=5.88 \mathrm{~cm}$. Measures are taken every hour starting at time $T_{1}=6 h$ for seven times. We found

$$
\mathrm{g}=-(.524, .429, .345, .264, .195, .181, .168) \text {. }
$$

We solved the singular system and found in particular that $c(\mathcal{A})=\lambda_{M} / \lambda_{m} \approx 4.932$ which is quite acceptable. The generalized solution $w^{\dagger}$ of the inverse problem (the solid line in the figure 15looks rather irregular, being essentially a linear combinations of characteristic functions. The dashed curve is obtained by "smoothing" $w^{\dagger}$ around a $\beta \delta^{2}$-type curve with $\beta \approx 2.2 \mathrm{~cm}^{-1} \times \mathrm{s}^{-1}$; a comparison between the latter and (2) (the dotted line) reveals the presence of retarding effects (due for instance to the non-spherical shape of particles, mutual interactions etc.).

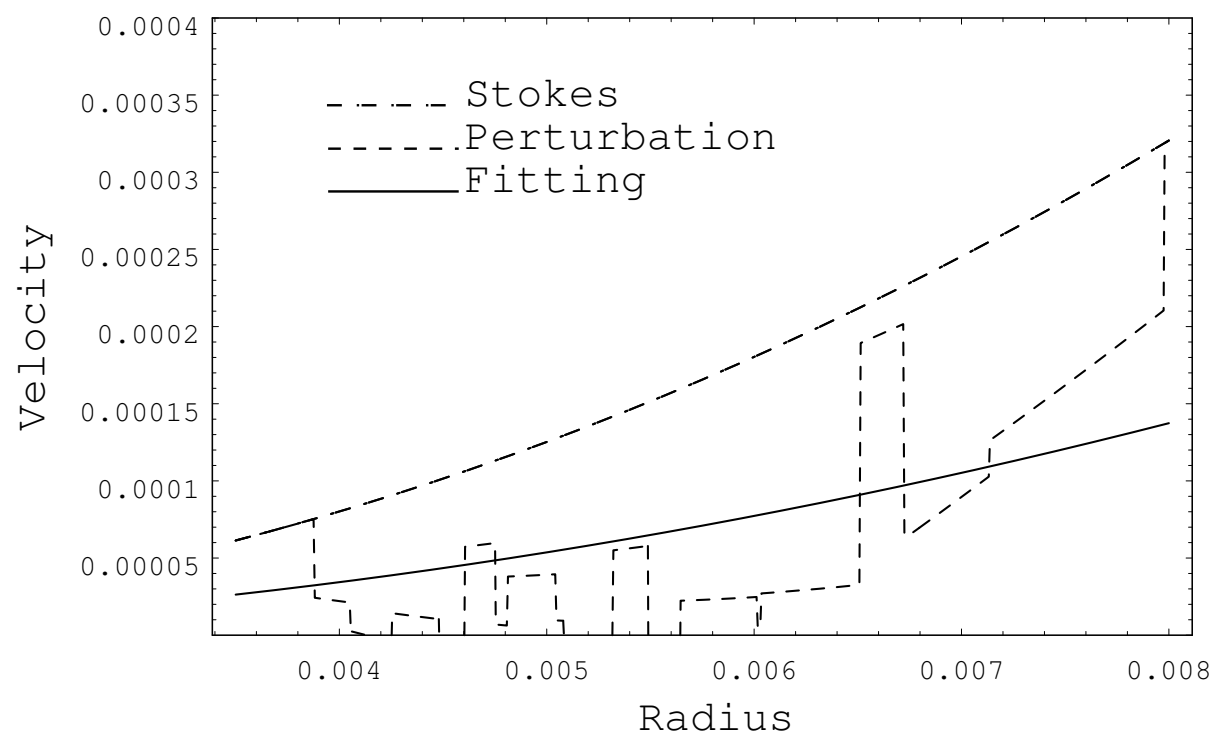

Figure 15: The generalized solution of the inverse problem compared with the Stokes's solution and a $\delta^{2}$-type curve fitting the available data points

${ }^{7}$ All experiments to collect the necessary data were worked out at Snamprogetti research facility at Fano (Italy) 


\section{LIST OF SYMBOLS:}

$\mathbf{v}_{s}$ : sedimentation velocity

$\delta$ : particle radius

$\delta_{0}$ : particle critical settling radius

g: gravity acceleration

$\rho_{s}:$ particle density

$\rho_{l}$ : liquid density

T: Cauchy stress tensor

$\mathbf{n}$ : outward unit normal to a regular surface

$\eta$ : Newtonian fluid viscosity

$\mathcal{R} e$ : Reynolds number

$\tau:$ shear stress

$\dot{\gamma}$ : shear rate

$\tau_{0}$ : yield stress (Bingham model)

$\eta_{B}$ : plastic viscosity (Bingham model)

$\mathcal{S}$ :the mass of settling particles present (at given time) in a observation cell of a rotational viscometer

$\frac{d p}{d x}$ : the constant pressure gradient $(<0)$ i a pipeline

$V_{x}(r)$ : fluid velocity profile in a pipeline,

$R$ : pipeline inner radius

\section{References}

[1] Acharya, A., Mashelkar, R.,A., Ulbrecht, J., Flow of inelastic and viscoelastic fluids past a sphere I. Drag coefficient in creeping and boundaty-layer flows Rheol. Acta, 15, pp. 454-470, 1976

[2] Adachi, K., Yoshioka, N., On creeping flow of a viscoplastic fluid past a circular cylinder Chem. Eng. Sci., 28, pp. 215-226, 1973

[3] Andres, V.T., Equilibrium and motion of a sphere in a viscoplastic fluid, Soviet Phis.Doklady, 5, pp. 723-726, 1960

[4] Ansley, R.,W., Smith, T.,N., Motion of Spherical Particles in a Bingham Plastic, AIChE Journal, 13, pp. 11931196, 1967

[5] Astarita, G., The Engineering reality of yield stress, J. Rheol., 34, pp. 275-277, 1990

[6] Beris, A.,N., Tsamopoulos, J.,A., Armstrong, R.,C., Brown, R.,A., Creeping motion of a sphere through a Bingham Plastic, J. Fluid Mech., 158, pp. 219-244, 1985

[7] Bertero, M., De Mol, C., Pike, E.,R., Linear Inverse Problems with Discrete Data. 1: General Formulation and Singular System Analysis Inverse Problems 1, pp. 301-330, 1985

[8] Bertero, M., De Mol, C., Pike, E.,R., Linear Inverse Problems with Discrete Data. 2: Stability and Regularization Inverse Problems 14, pp. 573-594, 1988

[9] Brenner, H., Dynamics of particles in a viscous fluid, Chem.Eng.Sci., 17, pp. 435-439, 1962

[10] Brookes, G.,F., Whitmore, R., L., Drag forces in Bingham plastics, Rheol.Acta, 8, pp. 472-480, 1969

[11] Carniani, E., Ercolani, D., Meli, S.,Pellegrini, L., Primicerio, M., Shear Degradation of Concentrated CWS in Pipeline Flow, Proc.13th Int. Conf. Slurry Technology, Denver, 1988

[12] Chhabra, R., P., Steady Non-Newtonian Flow About A Rigid Sphere, Encyclopedia of Fluid Mechanics, vol.1, pp. 983-1033, 1986

[13] Chhabra, R., P., Bubbles, Drops and Particles in Non-Newtonian Fluids CRC press, London 1992

[14] De Angelis, E. Modelli stazionari e non per fluidi di Bingham in viscosimetro e in condotta Ph.D. Thesis, University of Florence 1996 
[15] De Angelis, E., Fasano, A., Primicerio, M., Rosso, F. , Carniani, E., Ercolani, D., "Modelling sedimentation in CWS'in Hydrotransport 12 Proc. of the $12^{\text {th }}$ Int'l Conf. on Slurries Handling and Pipeline Transport, edited by C. A. Shook, MEP Pubbl. (1993), pp. 399-414.

[16] De Angelis, E., Mancini, A., A model for the dynamics of sediments in a pipe Mathematical and Computer Modelling, 25 pp. 65-78 (1997).

[17] De Angelis, E., Rosso, F., "A functional approach to the problem of evaluating the velocity for a population of particles settling in a liquid", Proc. of the $6^{\text {th }}$ European Consortium for Mathematics in Industry (1993), A. Fasano, M. Primicerio eds., B. G. Teubner 1994, pp. 191-198.

[18] De Angelis, E., Fasano, A., Primicerio, M., Rosso, F. , Carniani, E., Ercolani, D., "Sedimentation bed dynamics for fluids with yield stress in a pipe", Proc. of the $4^{\text {th }}$ Int. Conf. "Fluidodinamica Multifase nell'Impiantistica Industriale”, (1994), pp. 85-93.

[19] Dedegil, M., Y., Drag Coefficient and Settilng Velocity of Particles in Non-Newtonian Suspensions, Transaction of the ASME, 109, pp. 319-323, 1987

[20] Fasano, A., "A mathematical model for the dynamics of sediments in a pipeline", in Progress in Industrial Mathematics at ECMI 94, ed. by H. Neunzert, pp. 241-249, Weley and Teubner Publ. (1996)

[21] Fasano, A., Primicerio, M. “Modelling the rheology of a coal-water slurry", Proc. of the $4^{\text {th }}$ Europ. Consortium on Mathematics in Industry, edited by H. J. Wacker and W. Zulhener, B. G. Teubner and Kluwer Academic Publ. 1991, pp. 269-274

[22] Fasano, A., Primicerio, M., New results on some classical parabolic free boundary problems, Quart.Appl.Math., 38, pp. 439-460, 1990

[23] Fasano, A., Manni, E., Primicerio, M., Modelling the dynamics of fluidizing agents in coal-water slurries, Proc. of the International Symposium on Nonlinear Problems in Engineering and Science, ed. by S. XIAo and X. Hu, Science Press, Beijing, pp. 64-71 1992

[24] Fasano, A., Primicerio, M., Rosso, F. On Quasi-Steady Axisymmetric Flows of Bingham Type with Stress-Induced Degradation, Computing, 49, pp. 213-237, 1992

[25] Galdi, G., P. An introduction to the mathematical theory of the Navier-Stokes equations, Vol I: linearized stationary problems Springer Tracts in Natural Philosophy, 38, Springer Verlag, 1994

[26] Gianni, R., Pezza, L., Rosso, F., The constant Flow Rate Problem for Fluids with Increasing Yield Stress in a Pipe Theoretical and Computational Fluid Mechanics vol. 7, n. 6 (1995), pp. 477-493

[27] Leal, L., G., Particle motions in a viscous fluid Ann. Rev. Fluid Mech., 12, pp. 435-476, 1980

[28] Mancini, A., Evoluzione di profili di sedimentazione nel trasporto di sospensioni concentrate in condotta, Thesis Dept. Math. "U.Dini”, University of Florence, 1995

[29] Mancini, A., Evolution of sediment profiles in the transport of coal water slurries through a pipeline in Progress in Industrial Mathematics at ECMI 96, Ed. by M. Brøns, M. Bendsøe and M.P. Sørensen, Teubner, Stuttgart 1997, pp. 441-449

[30] Oseen, C., W., Über die Stokessche Formel und über eine Verwandte Aufgabe in der Hydrodynamik, Ark. Mat. Astron. Fys., 6, pp. 1-20, 1910

[31] Oseen, C., W., Neuene Methoden und Ergebnisse in der Hydrodynamik, Leipzig: Akademische Verlagsgesellshalt, 1927

[32] Primicerio, M., Dynamics of slurries, Proceedings of the 2nd Europen Consortium on Mathematics in Industry 1987, H. Neunzert ed., B.G. Teubner 1988, pp. 121-134

[33] Parrini, S., Moto stazionario di un fluido di Bingham in una condotta con sezione trasversale non circolare Thesis, Dept. Mathematics “Ulisse Dini”University of Florence, 1995

[34] Rosso, F., "On two inverse problems in hydrodynamics of non-Newtonian fluids" in Recent Advances in Mechanics of Structured Continua - 1993 Editors: M. Massoudi \& K. R. Rajagopal, AMD vol.160, ASME Publ., pp. 95-103

[35] Stokes, G., G., On The Effect of the Internal Friction of Fluids on the Motion of Pendulums, Trans.Cam.Phil.Soc., 9, pp. 8-27, 1851

[36] Terenzi, A., Carniani, E., Donati, E., Ercolani, D., Problems on nonlinear fluid dynamics in industrial plants this same volume. 
[37] Thomas, A., D., Settling of Particles in a Horizontally Sheared Bingham Plastic, First National Conf. on Rheology, Melbourne, pp. 89-92, 1979

[38] Tyabin, N., V. Some questions on the theory of viscoplastic flow of disperse systems, Colloid J. U.S.S.R., 15, pp. 153-159 1953

[39] Turian, R., M., Hsu, F., L., Avramidis, K., S., Sung, D., J., Allendorfer, R., K., Settling and Rheology of Suspensions of Narrow-Sized Coal particles, AIChE Journal, 38, pp. 969-987, 1992

[40] Valentik, L., Whitmore, R., L., The terminal velocity of spheres in Bingham plastics, Brit. J. Appl. Phis., 16, pp. 1197-1203, 1965

[41] Wildemuth, C., R., Williams, M., C., Viscosity of suspensions modeled with a shear-dependent maximum packing fraction, Rheol.Acta, 23, pp. 627-635, 1984

[42] Wilson, K., C., Hydrotransport 1 to 12, better by the dozen, opening address Hydrotransport 12, Int'l Conference on Slurry Handling and Pipeline Transport, Ed. by C.A. Shook, BHR Group Conference Series, Publication No. 6, Mechanical Engineering Publication Limited, London, 1993

[43] Yoshioka, N., Adachi, K. and Ishimura, H. On creeping flow of a viscoplastic fluid past a sphere Kagaku Kogaku, 10, pp. 1144-1152, 1971, (in Japanese) 